Documents pour l'histoire des techniques

Nouvelle série

20 | 2e semestre 2011

L'expérimentation « en plein air » ou " grandeur nature ": Une pratique scientifique au service de l'action (XIX'- $\mathrm{XX}^{\mathrm{e}}$ siècles)

\title{
Les collections ferroviaires du Conservatoire des arts et métiers, support de l'enseignement technique
}

The railway collections of the Conservatoire des arts et métiers, as educational models

Lionel Dufaux

\section{(2) OpenEdition}

Journals

Édition électronique

URL : http://journals.openedition.org/dht/1788

DOI : $10.4000 /$ dht. 1788

ISSN : 1775-4194

Éditeur :

Centre d'histoire des techniques et de l'environnement du Cnam (CDHTE-Cnam), Société des élèves du CDHTE-Cnam

Édition imprimée

Date de publication : 1 décembre 2011

Pagination : 147-166

ISBN : 978-2-9530779-7-1

ISSN : 0417-8726

\section{Référence électronique}

Lionel Dufaux, «Les collections ferroviaires du Conservatoire des arts et métiers, support de l'enseignement technique ", Documents pour l'histoire des techniques [En ligne], 20 | 2e semestre 2011, mis en ligne le 25 septembre 2012, consulté le 10 décembre 2020. URL : http:// journals.openedition.org/dht/1788; DOI : https://doi.org/10.4000/dht.1788 


\title{
Les collections ferroviaires du Conservatoire des arts et métiers, support de l'enseignement technique
}

\author{
Lionel Dufaux \\ Musée des arts et métiers - Cnam
}

\begin{abstract}
RÉSUMÉ
Les collections ferroviaires du Musée des arts et métiers se sont progressivement constituées depuis la fin des années 1830 et forment aujourd'hui un ensemble patrimonial exceptionnel. Ces collections présentent l'originalité de ne comporter aucun élément de matériel roulant (moteur ou tracté) préservé mais nombre pièces détachées et dessins probablement réunis dans une présentation complémentaire des cours de la chaire de Mécanique appliquée aux arts du Conservatoire des arts et métiers. Enrichies de plusieurs modèles réduits à caractère pédagogique (qui en forment la partie la plus remarquable), ces collections ont fait l'objet d'une réactualisation après la Seconde Guerre mondiale. Cette étude se propose d'apporter des éléments de réponse quant à la constitution de ce patrimoine unique tout en s'intéressant aux modalités d'acquisition et aux typologies présentes dans cette collection.
\end{abstract}

Résumés et mots clés en anglais sont regroupés en fin de volume, accompagnés des mots clés français

es collections ferroviaires du Musée des arts et métiers Lforment un ensemble patrimonial exceptionnel qui demeure pourtant largement méconnu'. Constituées progressivement depuis la fin des années 1830, ces collections recouvrent des typologies variées : modèles de démonstration, pièces détachées, dessins et photographies (soit près de cinq cents objets, plus de sept cents photographies et huit cent trente dessins).

L'actuelle exposition de ces collections dans le "domaine transports" du musée a été conçue pour présenter les principales étapes de l'histoire technique ferroviaire, depuis les premiers essais de l'ingénieur français Marc Seguin jusqu'aux prémices du train à grande vitesse. Pourtant, c'est dans une toute autre optique que cette collection a été réunie au Conservatoire des arts et métiers, tout au long du XIXe siècle et au début du XXe siècle ${ }^{2}$.

Les collections ferroviaires n'ont pas fait l'objet d'études complètes depuis l'édition du catalogue de la section « Transports sur rails » de 1952. La préparation

1 La très grande majorité des pièces de cette collection est conservée dans les réserves du musée, à Saint-Denis, où seuls les chercheurs peuvent y avoir accès.

2 Sur l'histoire du Conservatoire des arts et métiers, voir Alain Mercier, Un Conservatoire pour les arts et métiers, Paris, Gallimard, coll. « Découvertes ॥, 1993. de l'exposition temporaire Toujours plus vite ! Les défis du rail, conçue pour le Cnam Champagne-Ardenne en 2007 et développée pour le Musée des arts et métiers en 2009, a été l'occasion de nous intéresser de plus près à ce patrimoine unique. L'examen minutieux des objets et des dessins et les recherches menées pour documenter les œuvres nous ont conduit, une fois l'exposition temporaire ouverte, à approfondir nos recherches. Quelles étaient les motivations des acquisitions? Dans quel contexte les pièces sont-elles entrées au Conservatoire, établissement où les machines, modèles, dessins et livres servaient de support à la présentation des derniers perfectionnements industriels ? Comment et à qui étaient-elles présentées ? Ces collections servaientelles de support à un enseignement technique de haut niveau, destiné à former des techniciens qualifiés et des ingénieurs? Autant de questions auxquelles nous tentons de donner des éléments de réponse dans cette étude.

Un nouveau mode de transport, une nouvelle collection (de la Restauration à la fin du Second Empire) Quelques éléments généraux doivent être rappelés : si les premières circulations de véhicules guidés sur des voies remontent au XVle siècle, le chemin de fer en tant que système technique mécanisé date du tout début du XIXe siècle. 
En Angleterre, l'ingénieur Richard Trevithick conçoit en 1804 la première locomotive équipée d'une machine à vapeur. En 1813, George Stephenson imagine la Blücher, première locomotive aux roves adhérentes: désormais, les machines rouleront sur le sommet des rails, et non plus dans une rigole creusée dans le sol. La première ligne de chemin de fer ouvre en Angleterre en 1825 et, quatre ans plus tard, Stephenson remporte le concours de Rainhill où sa Rocket (Fusée) circule à près de $50 \mathrm{~km} / \mathrm{h}$.

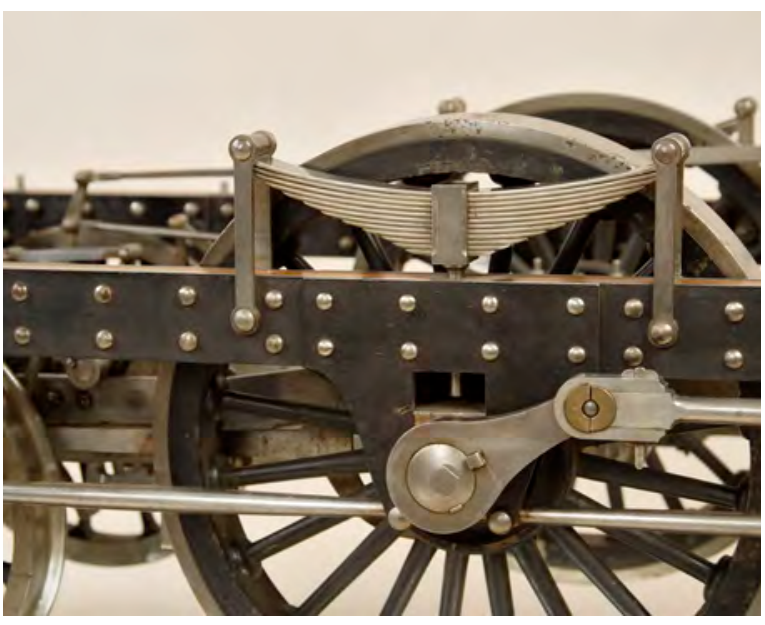

Châssis de locomotive Stephenson. Modèle au 1/5 réalisé par Eugène Philippe, 1838 (détail). Inv. 02549. (C) Musée des arts et métiers - Cnam / F. Botté

Une première remarque s'impose : ces événements, fondateurs de l'histoire des chemins de fer, ne sont quasiment pas représentés dans les collections du Conservatoire. La Rocket entre tardivement dans les collections (vers 1890), avec une reproduction dans le Portefeuille industriel (inv. 13571.2534). La locomotive de Stephenson y est représentée dans les collections du South Kensington Museum de Londres. Cette locomotive pionnière n'est entrée, quant à elle, qu'en 1862 dans les collections de ce musée, ancêtre du Science Museum³ . Dans les années 1860, en Angleterre, on cherche manifestement à célébrer la figure de certains inventeurs, notamment les pionniers du chemin de fer comme George et Robert Stephenson ${ }^{4}$. Cette

3 John Liffen, «The Patent Office Museum and the Beginnings of Railway Locomotive Preservation » in Michael Jonathan Taunton Lewis (dir.), Early Railways, Londres, Newcomen Society, t. 2, 2003 ; id., « Le Patent Office Museum, précurseur du Science Museum ॥, Musée des arts et métiers, La Revue, n 51/52, février 2010, pp. 56-67.

4 Voir à ce sujet Christine MacLeod, Heroes of Invention. dimension n'est pas visible en l'état dans les collections ferroviaires du Conservatoire, où une maquette de la locomotive de Marc Seguin de 1829 n'est acquise qu'en 1891 (inv. 12151).

En France, la première ligne (entre Saint-Étienne et Andrézieux, dans la Loire) est concédée le 26 février 1823. Mise en service le 30 juin 1827 avec une traction hippomobile, il s'agit de la première ligne de chemin de fer d'Europe continentale. Entre 1830 et 1832, la ligne de Saint-Étienne à Lyon est progressivement mise en service, bientôt complétée par la ligne d'Andrézieux à Roanne. Les premières lignes parisiennes (Saint-Germain-en-Laye - 1837, Versailles par la rive gauche de la Seine - 1839 et Versailles par la rive droite - 1840) seront représentées par des dessins du Portefeuille industriel ${ }^{5}$ (inv. 13571.757 ou 13571.692).

En 1834 sont acquis les premiers objets relatifs aux chemins de fer, une locomotive Stephenson et des wagons au 1/5 (inv. 04044). L'ensemble est réalisé par Eugène Philippe, constructeur de modèles réduits, à partir de la locomotive exposée dans les ateliers de la Fonderie de Chaillot en 1833 et dont César Nicolas Louis Leblanc, dessinateur au Conservatoire, donne les plans (inv. 13571.751). Ces modèles sont distingués, comme les productions de Philippe, par l'Exposition des produits de l'industrie française de 1834. En 1839 est inscrit à l'inventaire un modèle réduit du châssis d'une locomotive de type 120 par Eugène Philippe, d'après George Stephenson, permettant d'apprécier la transmission mécanique du mouvement (inv. 02549). II nous faudra déterminer si ces objets et les premiers dessins ont été utilisés comme un support avec les enseignements du Conservatoire ou s'ils ont servi dans le cadre d'expériences de laboratoire.

\section{Le chemin de fer dans les enseignements du Conservatoire des arts et métiers}

La formation de cheminots possédant un savoir-faire de qualité se révèle indispensable, notamment dans le cadre du transfert des connaissances des cheminots britanniques vers leurs homologues français.

Cette transmission peut s'opérer au sein des ateliers ferroviaires, mais également dans des écoles d'enseignement de haut niveau. C'est ainsi que l'ingénieur des Mines Auguste Perdonnet',

Technology, Liberalism and British Identity 1750-1914, Cambridge University Press, 2007, pp. 198-211.

5 Le numéro d'inventaire 13571 renvoie à la collection de dessins duConservatoirequiporte, depuislemilieuduXIX ${ }^{\circledR}$ siècle,lenomde «Portefeuille industriel».

6 Le dessin de la locomotive de Hawthorn du chemin de fer de Versailles-Rive gauche (inv. 13571.757) porte la signature de Perdonnet. 


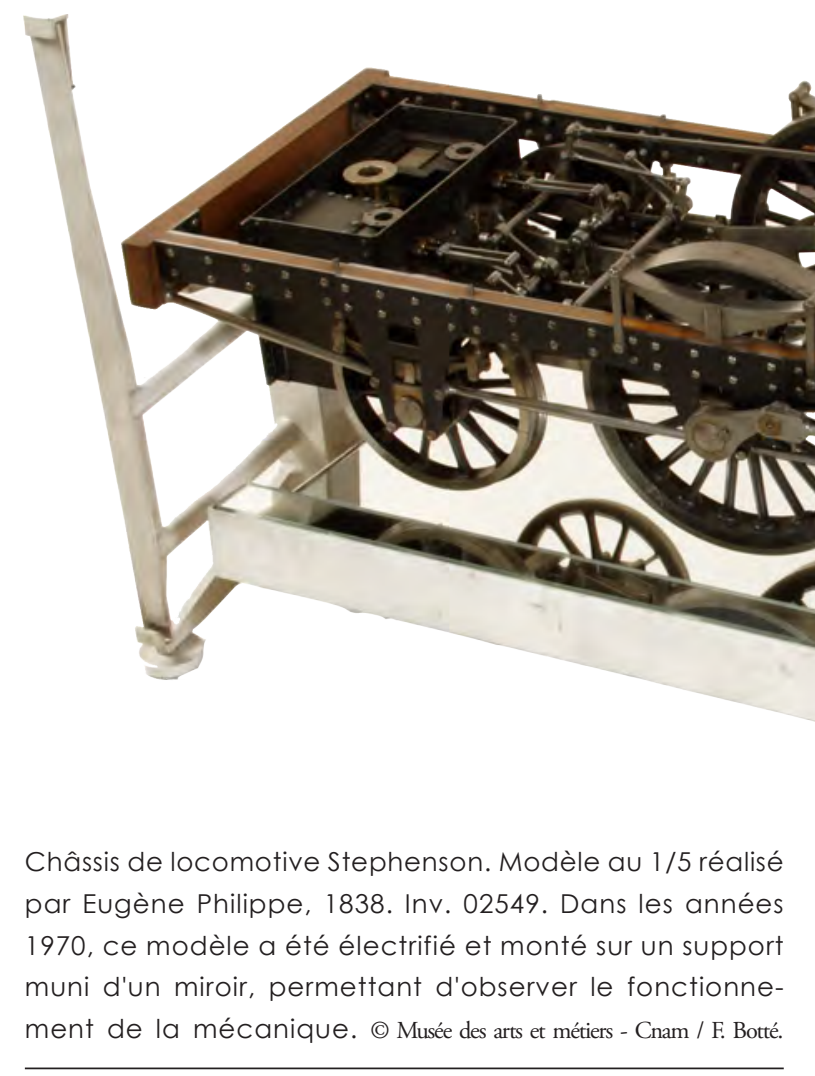

directeur du matériel de la Compagnie de Paris à Versailles-Rive gauche, ouvre un cours de chemin de fer à l'École centrale des arts et manufactures; en 1832, Joseph Minard enseigne des « notions [...] sur les chemins de fer ॥ à l'École des ponts et chaussées?. Au Conservatoire en revanche, l'examen des procès verbaux du Conseil de perfectionnement nous a montré qu'il n'existait pas de cours de chemin de fer. La question ferroviaire y est abordée d'une manière que nous pourrions qualifier de "périphérique".

\section{Le chemin de fer, objet économique}

En 1832, Jean-Baptiste Say proposait au conseil de perfectionnement du Conservatoire de présenter des modèles de machines ferroviaires ${ }^{8}$. Titulaire de la chaire d'économie industrielle, Say intervient à une époque où les systèmes techniques se complexifient. II perçoit le chemin de fer comme un mode de transports qui peut se substituer avantageusement aux routes et canaux, avec une forte augmentation des vitesses commerciales ${ }^{9}$. Des

7 Victorin Chevallier, "Notice nécrologique de M. Minard, inspecteur général des Ponts et Chaussées, en retraite 1), Annales des ponts et chaussées, $5^{e}$ série, †. II, deuxième semestre 1871, Paris, Dunod, 1871.

8 Procès-verbal du Conseil de perfectionnement du 19 novembre 1830.

9 Jean-Baptiste Say, Cours complet d'économie politique, $3^{\circledR}$ éd., Bruxelles, Dumont, 1836, p. 453.

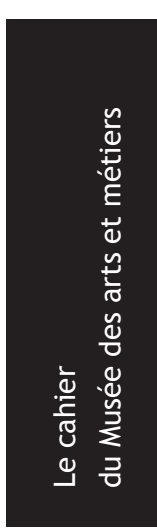

modèles pourraient montrer aux auditeurs parisiens ce qu'est le chemin de fer, cinq ans avant l'ouverture de la première ligne au départ de la capitale.

Le chemin de fer reste présent dans le cours d'Adolphe Jérôme Blanqui, quisuccède à Say en 1834 comme titulaire de la chaire d'économie politique ${ }^{10}$. Blanqui consacre trois leçons aux chemins de fer dans le cadre de la présentation des différentes « voies de communication $»^{11}$. Il expose les avantages du transport ferroviaire par rapport au roulage et indique que le chemin de fer constitue la meilleure solution pour le transport des hommes sur de longs parcours. Après une intéressante comparaison des situations française et britannique, Blanqui s'arrête sur la place que l'État doit tenir dans la construction, l'exploitation et les règlements des chemins de fer. C'est là un reflet des débats passionnés qui ont précédé la fameuse loi du 11 juin 1842 où ont été programmées les constructions des principales lignes radiales françaises et où le rôle de l'État a été défini par rapport à celui des compagnies exploitantes.

Le cours semble toutefois déconnecté des collections du Conservatoire que Blanqui ne mentionne nulle part dans son discours.

Le chemin de fer, la mécanique et la physique Certains observateurs déplorent, au début des années 1850, l'absence au Conservatoire d'un cours

10 Adolphe Jérôme Blanqui, Conservatoire des arts et métiers. Cours d'économie industrielle, 1836-1837, Paris, Angé, 1837. 11 Adolphe Jérôme Blanqui, Cours d'économie industrielle, 1838-1839, Paris, L. Mathias, 1839, pp. 425-472. 


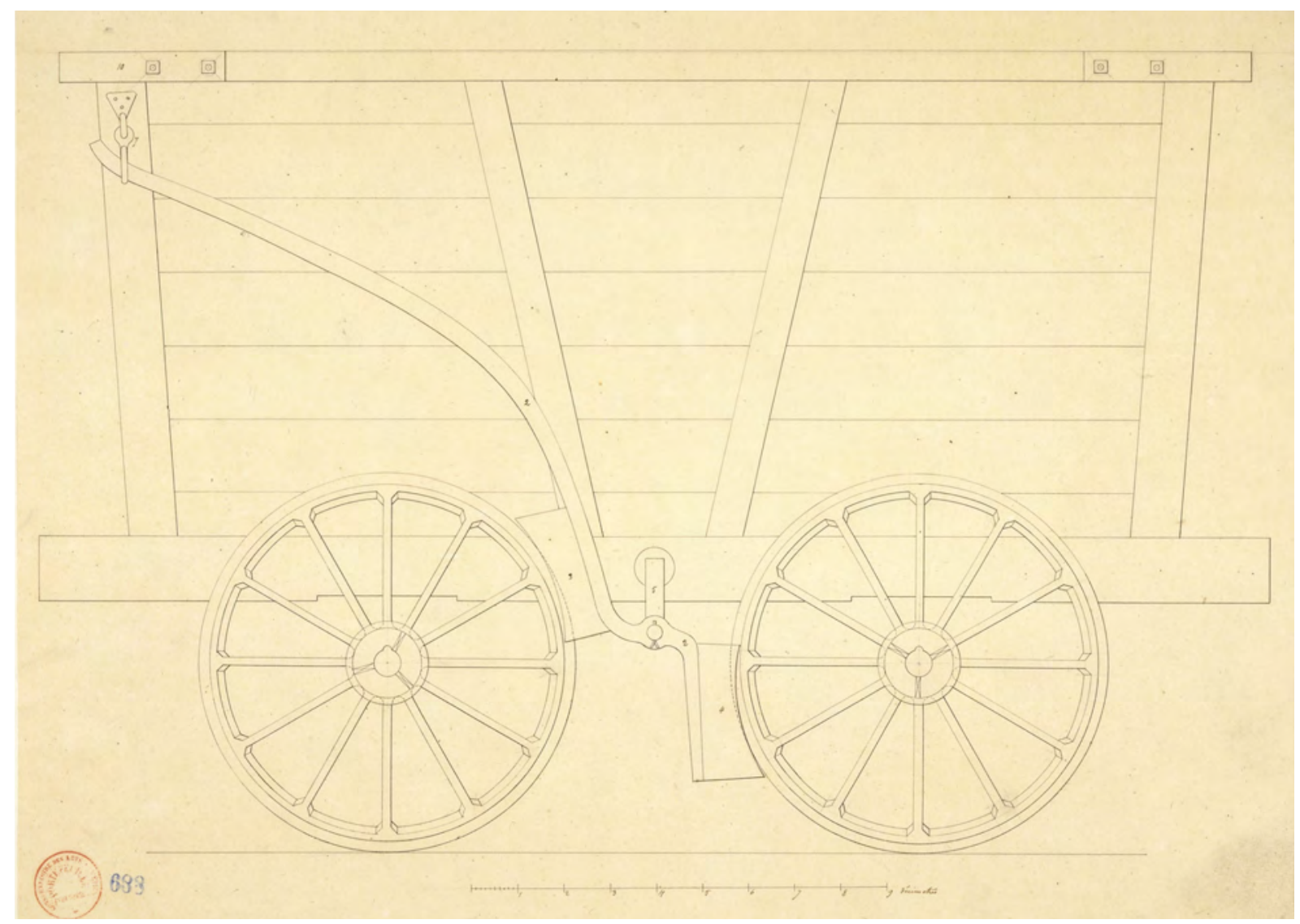

César Nicolas Louis Leblanc, chariots du chemin de fer de la Loire (détail). Calque contrecollé sur carton, vers 1830. Portefeuille industriel, inv. 13571.688/1. (c) Studio Photo Cnam.

" d'éducation spéciale pour les ouvriers des chemins de fer $\|\rangle^{12}$. Pourtant, la chaire de mécanique appliquée à l'industrie (devenue en 1851 mécanique appliquée aux arts) proposera une approche originale du chemin de fer comme application de la mécanique.

Arthur Morin, titulaire de la chaire entre 1839 et 1859, et son successeur Henri Tresca (entre 1859 et 1885) se sont servis de modèles de locomotives pour réaliser diverses expériences sur des moteurs à vapeur. Ils se sont, entre autres, intéressés aux questions relatives à la vaporisation et ont ainsi travaillé sur les différents types de chaudières, pour machines mobiles ou installations fixes, en bénéficiant parfois de la collaboration de l'industrie ferroviaire ${ }^{13}$. À cette époque, la collection s'enrichit de divers éléments relatifs à la production et à l'utilisation de la vapeur : tubes à fumée

12 Edmond Texier, Tableau de Paris, Paris, Paulin et Le Chevalier, 1853, p. 185

13 Arthur Morin et Henri Tresca, Mécanique pratique des machines à vapeur. La production de la vapeur, Paris, Hachette, 1863. (inv. 05718 et 05719), tiroirs pour distribution (inv. 05724), soupapes (inv. 05725 et 05726), l'ensemble entrant en 1853. Morin se sert également du chemin de fer pour aborder les questions de transmission et de transformation du mouvement. La transformation d'un mouvement alternatif de va-et-vient (produit par la machine à vapeur) en un mouvement rotatif (des roves) par le biais d'une bielle-manivelle est un fondamental de la technique de la traction ferroviaire ${ }^{14}$. Les pistons (inv. 06435 et 08020 à 08023) et modèles de distribution (inv. 02549, 03080, 07266, 08685 ) en sont de parfaites illustrations. Morin aborde aussi les forces physiques et les contraintes qu'elles exercent sur les chemins de fer, notamment lors des passages des virages (force centrifuge) et dans les phases d'accélération et de freinage ${ }^{15}$. II convient de mentionner les travaux de Morin, devenu entre-temps directeur du Conservatoire, au sujet de l'usure des rails en fer ${ }^{16}$. Nous n'avons pour le moment pu déterminer

14 Arthur Morin, Leçons de mécanique pratique. Notions géométriques sur les mouvements et leurs transformations, Paris, Hachette, 1861.

15 Arthur Morin, Leçons de mécanique pratique. Notions fondamentales de mécanique, Paris, Hachette, 1860.

16 « Note sur la substitution des rails en acier Bessemer aux rails en fer sur les parties les plus fatiguées des chemins de fer "), Annales du Conservatoire impérial des arts et métiers, $t$. VII, 
précisément si les objets entrés au musée ont servi dans le cadre des travaux du laboratoire expérimental de mécanique mis en place vers 1850 par Morin. Le dépouillement des Annales du Conservatoire des arts et métiers, entre 1861 et 1902, ne nous permet pas de l'affirmer, même si l'on y retrouve les procès-verbaux d'expériences menées sur des machines à vapeur.

\section{La " galerie dite des chemins de fer "}

Si les modèles de chemin de fer ont pu illustrer plusieurs cours, ils sont avant tout destinés à être exposés pour être vus du public. Une galerie, dite « des chemins de fer ", est aménagée vers 1840 au premier étage du corps central ${ }^{17}$. On peut y découvrir " l'établissement des rails et deux systèmes différents de locomotives [...] exécutés au cinquième $\|^{18}$. Cette salle se trouve à proximité des espaces consacrés à la géométrie descriptive et à la mécanique. Cette observation renforce l'idée que les collections ne se sont pas constituées à l'origine comme une série dévolve aux transports mais bien aux applications concrètes de la mécanique. En 1851, la collection compte dix-neuf objets ferroviaires que nous avons tenté d'identifier par comparaison avec la main courante du musée :

« 1. Locomotive à quatre roves, avec son tender, d'après Stephenson. [...] [inv. 04044]

2. Locomotive à six roues, dont le dessus est supprimé afin de laisser voir le mécanisme. [inv. 02549]

3. Train de tender mixte à six roves, en usage sur le chemin de fer de Lyon.

4. Waggon [sic] pour les marchandises. [inv. 04045]

5. Waggon pour la houille. [inv. 04046]

6. Waggon pour les fers. [inv. 04047]

7. Waggon à bagages avec application du frein de Noseda. [inv. 03213]

8. Détente variable de Meyer, appliquée à une locomotive. [inv. 03080]

9. Tuyau alimentaire pour emplir les tenders. [inv. 02550]

10. Sifflet de locomotive de grandeur naturelle.

11. Modèle d'une bielle de locomotive. [inv. 02897]

12. Modèle d'un excentrique de locomotive. [inv. 02896]

13. Modèle de chemin de fer montrant l'établissement des rails. [inv. 04041]

14. Changement de voie. [inv. 04042]

15. Tourne-rail.

16. Tourne-rail.

1866-1867, pp. 474-485.

17 Arthur Morin, Conservatoire des arts et métiers. Catalogue des collections, Paris, Guiraudet et Jouaust, 1851, p. XXXIII. 18 Édouard Charton, Le Magasin pittoresque, 1843, p. 190.
17. Indicateur du temps de la marche des convois, donnant le temps passé aux stations par l'excès de la durée totale du parcours sur celle de la marche. [inv. 03093]

18. Indicateur du passage des trains sur la voie, donnant le temps écoulé entre les passages de deux convois consécutifs, par Paul Garnier. [inv. 03092]

19. Appareil destiné à montrer l'une des causes de déraillement des locomotives ${ }^{19}$. 11

Nous pouvons d'ores et déjà remarquer trois éléments au sujet de cette collection primitive :

- Le Conservatoire expose en 1851 des locomotives dépassées : la locomotive à quatre roves de Stephenson et la locomotive à six roves sont déjà anciennes et ont en grande partie disparu des lignes françaises à l'époque. Elles sont en revanche très intéressantes d'un point de vue didactique, puisqu'elles permettent de montrer les principes de fonctionnement des locomotives à vapeur.

- A contrario, certains objets illustrent de récentes innovations, comme la détente variable de Meyer, qui fait l'objet de plusieurs communications dans le Bulletin de la Société d'encouragement pour l'industrie nationale. Meyer recevra d'ailleurs un prix de 4000 francs et une médaille d'or pour son invention ${ }^{20}$.

- Enfin, ces collections reflètent les pratiques de l'époque, par exemple l'espacement des trains par le temps et non par la distance pour assurer leur sécurité. Jusqu'au début des années 1880, les règlements imposaient un intervalle de temps entre deux trains (en principe, une dizaine de minutes). Cet intervalle était fixe, que la voie soit libre ou non, ce qui rendait l'exploitation particulièrement dangereuse. Progressivement, la notion d'espacement par la distance sera privilégiée : désormais, ce n'est que lorsque le voie sera libre qu'un train pourra s'y engager. Ces nouvelles pratiques ne seront possibles que grâce au perfectionnement des dispositifs de sécurité et au développement de la télégraphie ${ }^{21}$.

\section{Modèles et objets}

Modèles réduits de locomotives

Avec ses collections, le Conservatoire offre une vision élargie du premier système technique ferroviaire (1820-1870). Certains des modèles sont à rapprocher

19 Arthur Morin, Conservatoire des arts et métiers. Catalogue des collections, op. cit., pp. 189-190.

20 Bulletin de la Société d'encouragement pour l'industrie nationale, $47^{\mathrm{e}}$ année, $\mathrm{n}^{\circ} \mathrm{DXXIII,} \mathrm{p.} 664$.

21 Les collections télégraphiques du Musée des arts et métiers sont en ce sens très intéressantes : on y trouve plusieurs modèles de télégraphes mis au point pour renforcer la sécurité ferroviaire, comme un dispositif Breguet (inv. 10083, entré en 1858). 


\section{Les expériences localisées : le chemin de fer atmosphérique de Saint-Germain}

Les collections s'enrichissent par ailleurs d'éléments relatifs à certaines expérimentations isolées. C'est par exemple le cas du « chemin de fer atmosphérique de Saint-Germain-en-Laye » auquel une série de dessins (inv. 13571.692) est consacrée au moment de l'ouverture de cette courte ligne. Pour atteindre la terrasse de Saint-Germain, il fallait gravir une pente de $35 \mathrm{~mm} / \mathrm{m}$, infranchissable pour les locomotives de l'époque. La ligne nécessitait également la construction de plusieurs ponts, dont un sur la Seine, d'un tunnel sous la terrasse du château et d'une tranchée. Pour pallier ces problèmes techniques et financiers, la compagnie proposa de construire un chemin de fer atmosphérique. Ce système, testé en Irlande, permettait de tracter des convois à l'aide de pompes à vide. Les travaux commencèrent le 15 avril 1845, sous la direction d'Eugène Flachat, et furent achevés deux ans plus tard, jour pour jour. Huit cent cinquante tuyaux en fonte de $63 \mathrm{~cm}$ de diamètre formaient un tube fermé, à moitié enterré, dans lequel coulissait un piston portant une tige de connexion. Un wagon directeur, relié à cette tige, assurait la traction du convoi. Une pompe pneumatique, installée à SaintGermain, permettait de faire le vide dans le tube en seulement quatre minutes, la pression atmosphérique permettant de mouvoir le convoi. Le fonctionnement du chemin de fer atmosphérique demeurait toutefois compliqué et aléatoire, marquant le caractère expérimental de ce système. En 1858, les freins lâchèrent et le convoi percuta une locomotive en stationnement au Pecq. Trois personnes trouvèrent la mort dans cet accident. Finalement, deux ans plus tard, le 3 juillet 1860, le chemin de fer atmosphérique fut abandonné, de nouvelles locomotives, plus puissantes, tractant les rames jusqu'à Saint-Germain.

Plusieurs photographies du fonds Hovel (inv. 20627, 20628 et 20630) illustrent le fonctionnement de cette ligne "atmosphérique ", ainsi que des dessins plus spécifiquement consacrés aux systèmes d'attelage (inv. 13571.681). Voir Lionel Dufaux, "Voyage ferroviaire en banlieve ouest ", Musée des arts et métiers, La revue, $n^{\circ} 48$, octobre 2008, p. 55-62.

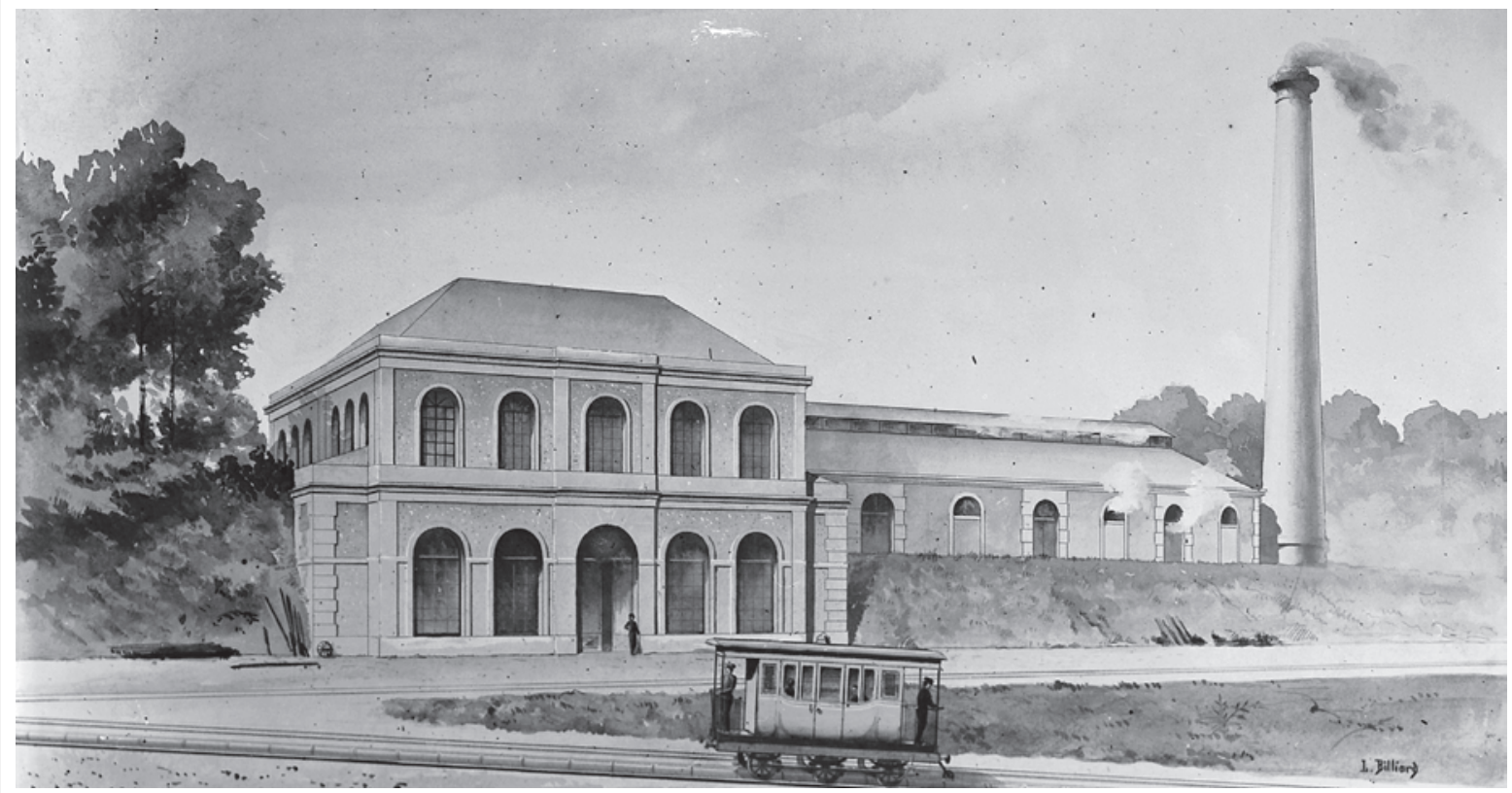

Les bâtiments des machineries du chemin de fer atmosphérique à Saint-Germain-en-Laye, vers 1847. Reproduction d'un dessin de Louis Billard. Tirage photographique positif réalisé à partir d'un négatif au gélatino-bromure d'argent sur plaque de verre, fin du XIXe siècle. Inv. 20628-0011-001. (c) Musée des arts et métiers - Cnam / M. Favareille.

des dessins du Portefeuille industriel (par exemple la locomotive Stephenson, inv. 13571.751 et 752 , ou les wagons à marchandises, inv. 13571.687 et 688).

Cette première période est marquée par les travaux pour faire fonctionner une locomotive (c'està-dire la juxtaposition d'une machine à vapeur et d'un châssis à l'aide d'un dispositif de transformation et de distribution de l'énergie). Le point de départ de l'exploitation efficace des chemins de fer est la production de vapeur en quantité suffisante grâce à la chaudière tubulaire. Le modèle réduit (inv. 17397) d'une chaudière de Marc Seguin permet d'apprécier, 
grâce à une coupe, le faisceau tubulaire intérieur. La date d'entrée de 1827, mentionnée dans le catalogue des collections de 1905 et la main courante, semble sans fondements ${ }^{22}$.

Les collections vont régulièrement s'enrichir de modèles réduits de locomotives. Un décalage de plusieurs années peut exister entre la conception d'un type de locomotive et l'entrée du modèle réduit dans les collections du Conservatoire.

En 1851, un modèle de locomotive Stephenson de type 111, conçu par Clair pour la démonstration, est acquis après l'Exposition universelle de Londres (inv. 04601). Plusieurs séries de dessins complètent ce modèle : locomotive $\mathrm{n}^{\circ} 42$ du chemin de fer du Nord par Desrosne et Cail (inv. 13571.756), locomotive Hawthorn du chemin de fer de Paris à VersaillesRive Gauche (inv. 13571.757), locomotive $\mathrm{n}^{\circ} 9$ du chemin de fer de Paris à Rouen par Alcard et Buddicom (inv. 13571.759), locomotive Meyer et Cie (inv. 13571.762, dessins d'Armengaud aîné), locomotive du Great Western Railway (inv. 13571.1520, entrée à la suite de l'Exposition universelle de 1862, à Londres) ${ }^{23}$.
Les "pièces détachées » des locomotives à vapeur Les travaux considérables menés par les ingénieurs sur les locomotives à vapeur pour gagner en puissance et en vitesse au cours de la période sont visibles à travers les très nombreuses "pièces détachées " entrées dans les collections. Si une présentation de « modèles complets " de locomotives s'avère plus didactique pour un public de non spécialistes, l'exposition de pièces détachées rend compte de manière plus précise des avancées techniques dans les domaines de la mécanique et de la thermodynamique (avancées que l'on retrouve dans les cours de la chaire de mécanique appliquée aux arts).

\section{L'alimentation en eau}

Aux pompes à eau (inv. 02552), largement employées pour remplir les chaudières des locomotives, on va préférer l'injecteur (plus fiable et utilisable lors de l'arrêt des locomotives), inventé par Giffard en 1858 (modèle Friedman, inv. 08656). Les réchauffeurs, apparus plus tardivement, portent la température de l'eau à $100^{\circ} \mathrm{C}$ en se servant de la vapeur d'échappement de la

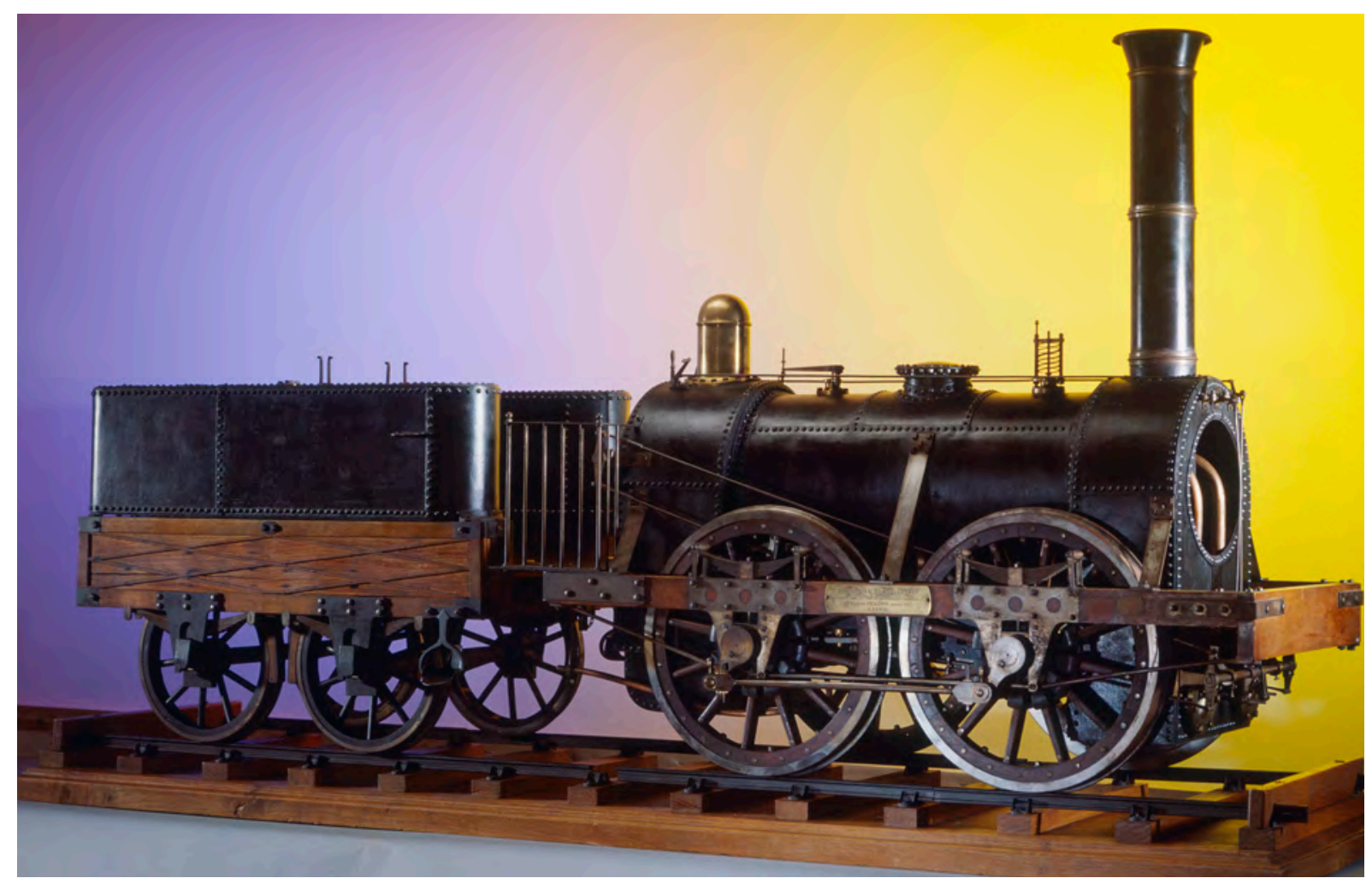

22 Jacques Payen, La Machine locomotive en France, des origines aux milieu du XIXe siècle, Lyon, Éditions du CNRS / Presses universitaires de Lyon, 1988, p. 220.

23 Sur les expositions universelles, voir Marie-Sophie Corcy, "Exposer l'invention », Musée des arts et métiers, La revue, $n^{\circ} 51 / 52$, p. 78.
Locomotive de George Stephenson. Modèle au 1/5 d'Eugène Philippe, 1833. Inv. 04044.

(C) Musée des arts et métiers - Cnam / S. Pelly. 

Page ci-contre : la salle des machines en mouvement, créée par le général Morin, permettait aux visiteurs de découvrir le fonctionnement d'équipements. Le Conservatoire pouvait également la mettre au service des industriels pour réaliser des expériences. Gravure extraite de La Nature, $2^{\text {ème }}$ semestre 1880.

(c) Cnum - Conservatoire numérique des arts et métiers (www.cnum.fr). Bibliothèque du Cnam.

locomotive, ce qui offre une économie de carburant non négligeable (l'eau étant déjà chaude quand elle arrive dans la locomotive).

\section{La distribution de la vapeur}

La distribution est l'étape pendant laquelle la vapeur quitte la chaudière pour travailler dans un cylindre où elle va agir sur un piston qui va mouvoir les roves. La distribution transforme le mouvement alternatif de va-et-vient, produit par le piston, en un mouvement rotatif. La collection du Conservatoire possède plusieurs modèles de distributions qui soulignent diverses améliorations (notamment la limitation des frottements pour optimiser le travail) : distribution Meyer (inv. 03080), Gooch (inv. 08685), Polonceau (modèle à double coulisse, inv. 07266) ; modèle de robinet à papillon (inv. 02551). Le Portefeuille industriel renferme plusieurs séries de dessins complémentaires : distribution à mouvement elliptique (inv. 13571.1963), mécanismes de distribution des locomotives " La Gironde » et « Le Saint-Germain » (inv. 13571.760), mécanismes de distribution à détente variable (inv. 13571.1755) ou encore dispositifs pour marche à contre-vapeur (inv. 13571.1821).

\section{Foyers, chaudières et échappements}

Dans son ouvrage Mécanique pratique des machines à vapeur, Arthur Morin listait les principales familles de chaudières, parmi lesquelles figuraient les chaudières tubulaires pour locomotives ${ }^{24}$. Les collections du Conservatoire se sont enrichies de très nombreux éléments constitutifs du système de production de la vapeur à l'intérieur des locomotives. On trouve ainsi les tubes à fumée (à l'intérieur desquels circule l'air qui va chauffer l'eau de la chaudière), entrés au moment où Morin dispense ses cours de mécanique (inv. 05718 et 05719, acquis en 1853). Les modèles d'échappement entreront sur le tard dans les collections, mais signalons les planches du Portefeuille relatives à « divers systèmes de régulateur et d'échappement» (inv. 13571.754).

\section{Mécanismes divers}

Le chemin de fer repose sur des dispositifs mécaniques parfois très sophistiqués. Le châssis de

24 Arthur Morin et Henri Tresca, op. cit. la locomotive 120 Stephenson (inv. 02549) en est une belle illustration. Les collections comptent également plusieurs éléments provenant de pistons (inv. 08020 à 08023), des tiroirs (inv. 05724), des bielles et des excentriques pour distribution (inv. 02896), mais aussi des dessins de dispositifs d'attelages (inv. 13571.681), de roues (inv. 13571.680) et d'essieux (inv. 13571.805). Les modèles et dessins consacrés aux freins sont nombreux et recouvrent plusieurs dispositifs, comme le frein à patins, le frein à vide, le frein à air comprimé ou encore le frein continu.

\section{Expansion ferroviaire et redéploiement des collections}

François Caron a montré que le chemin de fer était devenu, à partir des années 1870, un modèle d'exploitation rationnel. «Entre 1870 et 1914, les transformations du système ont été le résultat d'une concertation permanente [...] entre les services des compagnies ferroviaires [...]. La prise de conscience collective - par une nouvelle génération d'ingénieurs - de la gravité des dysfonctionnements et des insuffisances du système antérieur a débouché sur une vision cohérente et rationalisée de l'ensemble de l'exploitation et des services ${ }^{25}$. " Après 1870, les lignes principales, partant de Paris et rejoignant les frontières du pays, sont toutes achevées. Les concentrations opérées sous le Second Empire ont conduit à la création d'entreprises très importantes, se répartissant l'exploitation du réseau ferroviaire national selon des grandes zones géographiques. Afin de garantir une exploitation rentable, il a fallu considérablement moderniser le système ferroviaire initial dont le matériel roulant, l'infrastructure ou encore les règles de sécurité n'étaient pas toujours adaptés.

On va donc assister, entre 1870 et la Seconde Guerre mondiale, à une phase intense de modernisation des chemins de fer français qui vont se démarquer, de par leur matériel et leurs infrastructures, du modèle britannique initial. Cette évolution reflète la mise à jour des collections dans les domaines moteurs du développement industriel par Aimé Laussedat, directeur du Conservatoire entre 1881 et $1900^{26}$.

En 1886, l'ingénieur des Ponts et Chaussées Joseph Hirsch succède à Tresca à la chaire de mécanique appliquée aux arts. Titulaire de la

25 François Caron, «Le chemin de fer, un système technique » in Toujours plus vite ! Les défis du rail, Paris, La Vie du Rail / Musée des arts et métiers, 2009, p. 12-25 et plus particulièrement p. 15. 26 Claudine Fontanon et André Grelon (dir.), Les Professeurs du Conservatoire national des arts et métiers, Paris, INRP/ Cnam, 1994, t. 1, p. 43. 


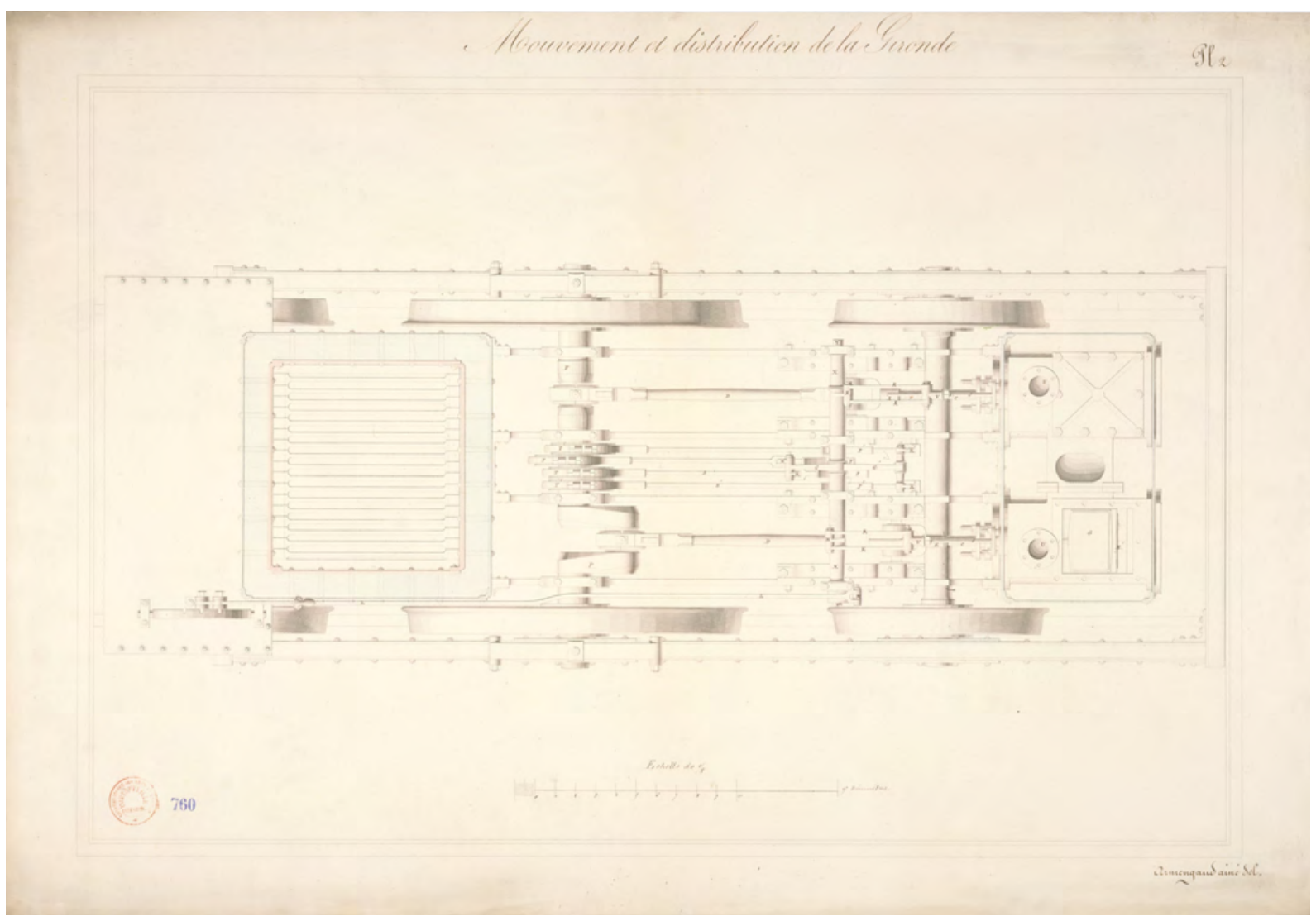

Jacques Eugène Armengaud dit Armengaud Aîné, Mouvement et distribution de la Gironde et Distribution et détente de la machine $S^{\dagger}$ Germain. Lavis sur traits de crayon et d'encre, vers 1840. Portefeuille industriel, inv. 13571.760/2 et 4. @ Studio Photo Cnam.

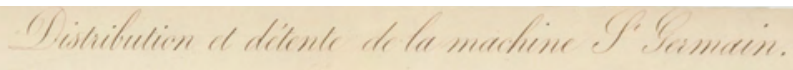

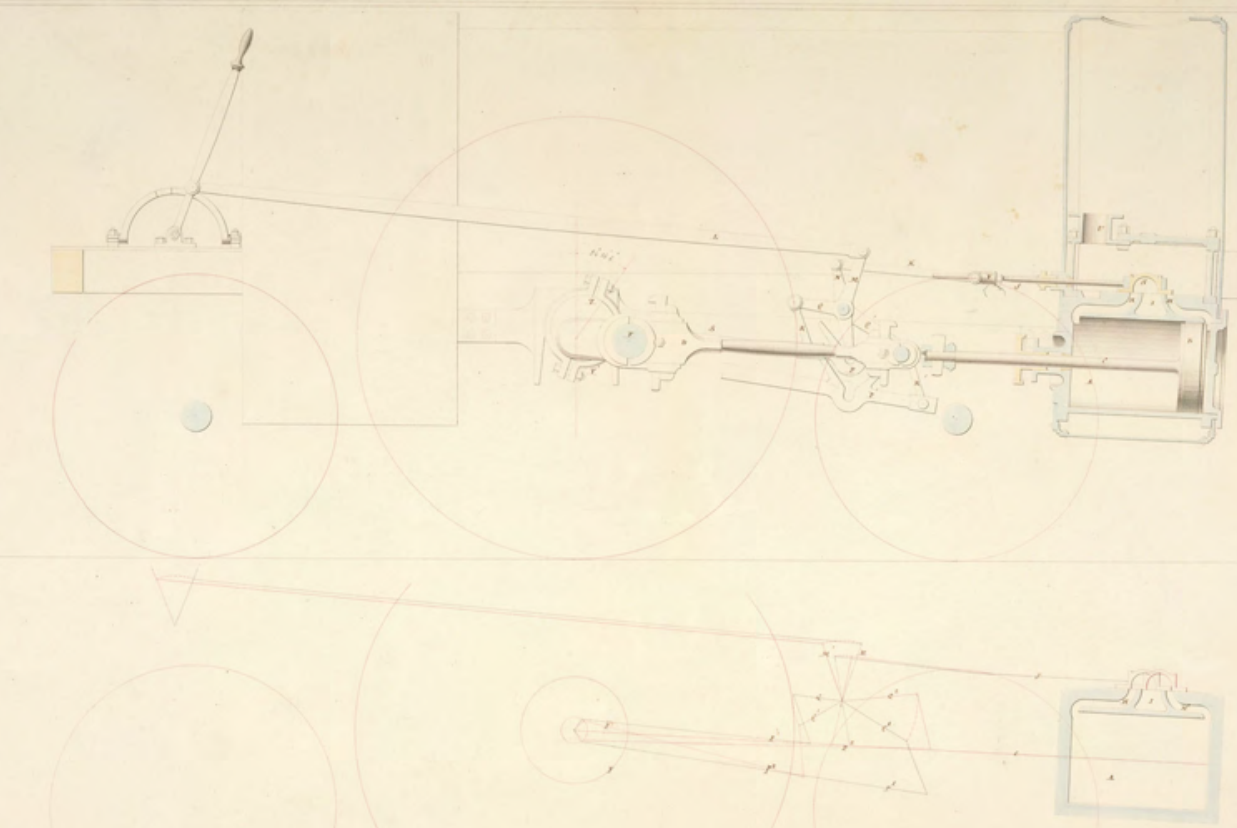


Locomotive Crampton. Modèle au 1/5 d'Eugène Cuvelier, 1858. Inv. 13767.

(๑) Musée des arts et métiers - Cnam / M. Favareille.

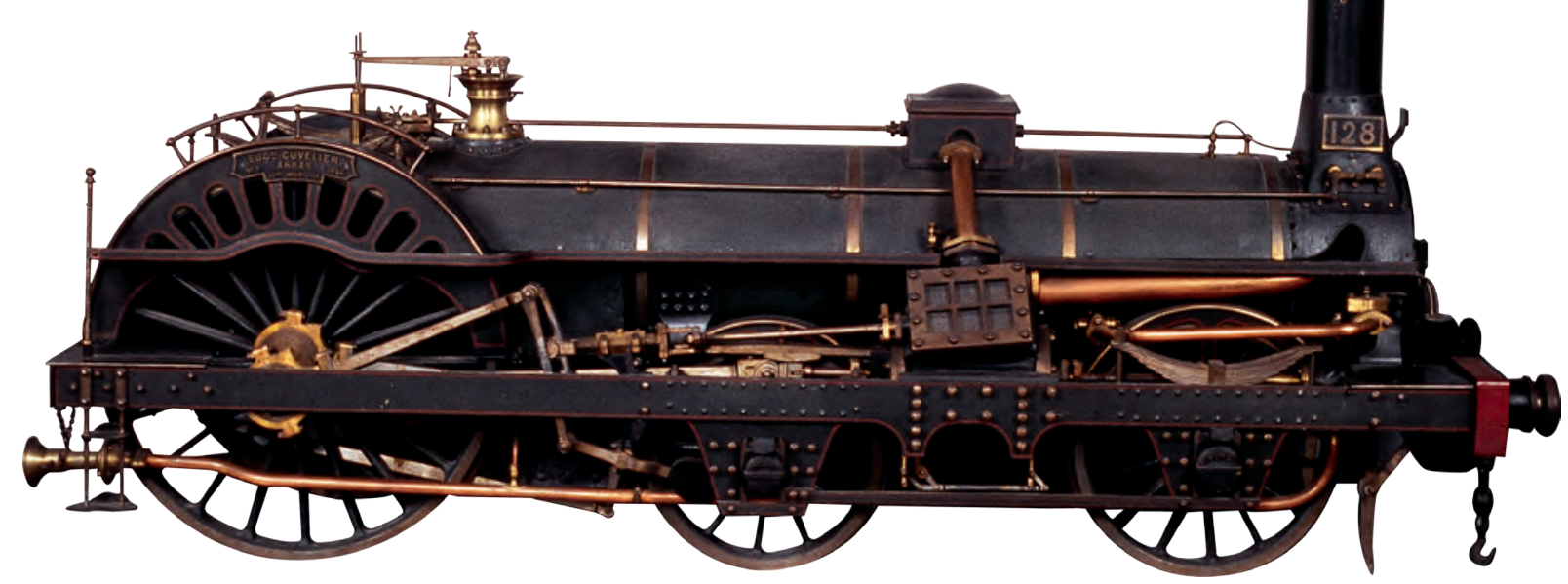

chaire des machines à vapeur à l'École des ponts et chaussées, Hirsch est un spécialiste de la thermodynamique. Dans ses cours du Conservatoire, il propose plusieurs séances sur " la machine à vapeur appliquée aux transports $11^{27}$. C'est d'ailleurs en qualité de professeur au Conservatoire qu'il prononce une conférence, "La locomotive moderne ", devant la Société industrielle de l'Est, en $1896^{28}$.

Le successeur ${ }^{29}$ de Joseph Hirsch, Édouard Sauvage, est un spécialiste de la traction ferroviaire. Ingénieur aux compagnies du Nord, de l'Est et de l'Ouest, il est l'auteur de La Machine locomotive, manuel de vulgarisation destiné à la formation des personnels, qui connut un succès considérable entre 1900 et 1950, s'adaptant aux évolutions techniques ${ }^{30}$. Reconnu internationalement pour la qualité de ses travaux, Sauvage a ouvert la voie aux immenses progrès dans les domaines de la mécanique et de la thermodynamique ${ }^{31}$ qui

27 Bulletin de la Société d'encouragement pour l'industrie nationale, 1897, 96 année, 5e série, t. 2, p. 1536.

28 Joseph Hirsch, "La locomotive moderne ", Annales du Conservatoire des arts et métiers, $2^{e}$ série, †. 9, p. 208.

29 En 1907, la chaire de mécanique appliquée aux arts prend le nom de chaire de machines. Voir Georges-Albert Boutry (et al.), Cent cinquante ans de haut enseignement technique au Conservatoire national des arts et métiers, Paris, Cnam, 1970. 30 La Machine locomotive. Mémoires de la Société des ingénieurs civils, vol. 72, 1er semestre 1899, p. 1086

31 Sauvage est le premier à intégrer des notions de thermodynamique dans les cours de la chaire de mécanique appliquée aux arts. Voir Konstantinos Chatzis, "L'enseignement des machines au Cnam ", in Claudine toucheront les chemins de fer pendant l'entredeux-guerres, entre autres en la personne d'André Chapelon ${ }^{32}$.

Le chemin de fer dans la Grande Galerie de mécanique Le réaménagement du premier étage du Conservatoire entraîne une réorganisation des collections ferroviaires, qui intègrent vers 1897 la « Grande Galerie de mécanique » (salle 24), à droite de l'escalier d'honneur ${ }^{33}$. Les collections de dessins sont quant à elles présentées au deuxième étage, dans la salle $53^{34}$.

\section{Les modèles de locomotives}

Les modèles de locomotives " complets » offrent une vision comparée de l'histoire ferroviaire et de l'innovation, sans doute destinée à un assez large public ${ }^{35}$. Au milieu du

Fontanon (dir.), Histoire de la mécanique appliquée. Enseignement, recherche et pratiques mécaniciennes en France après 1800, Paris, ENS Éditions / SFHST, coll. « Cahiers d'histoire et de philosophie des science $11, n^{\circ} 46,1998$, p. 23.

32 André Chapelon, ingénieur centralien, a contribué à perfectionner les locomotives des compagnies d' Orléans et du Nord. Voir en particulier Bernard Escudié, Jean Gréa et JeanMarc Combe, Thermodynamique et locomotives à vapeur : l'œuvre d'André Chapelon, 1892-1978, Éditions du CNRS, 1989. 33 |l s'agit de l'actuelle galerie dévolue aux collections du " domaine mécanique » du musée.

34 Conservatoire national des arts et métiers. Catalogue des collections, Paris, 1905.

35 Les modèles réduits du Conservatoire ont généralement été construits par les grands fabricants spécialisés dans ce genre d'outils : Eugène Philippe, Pierre Clair, Eugène Cuvellier, Jules Digeon. Voir entre autres Louis André, " Les modèles d'Eugène Philippe dans les collections du Conservatoire ", 


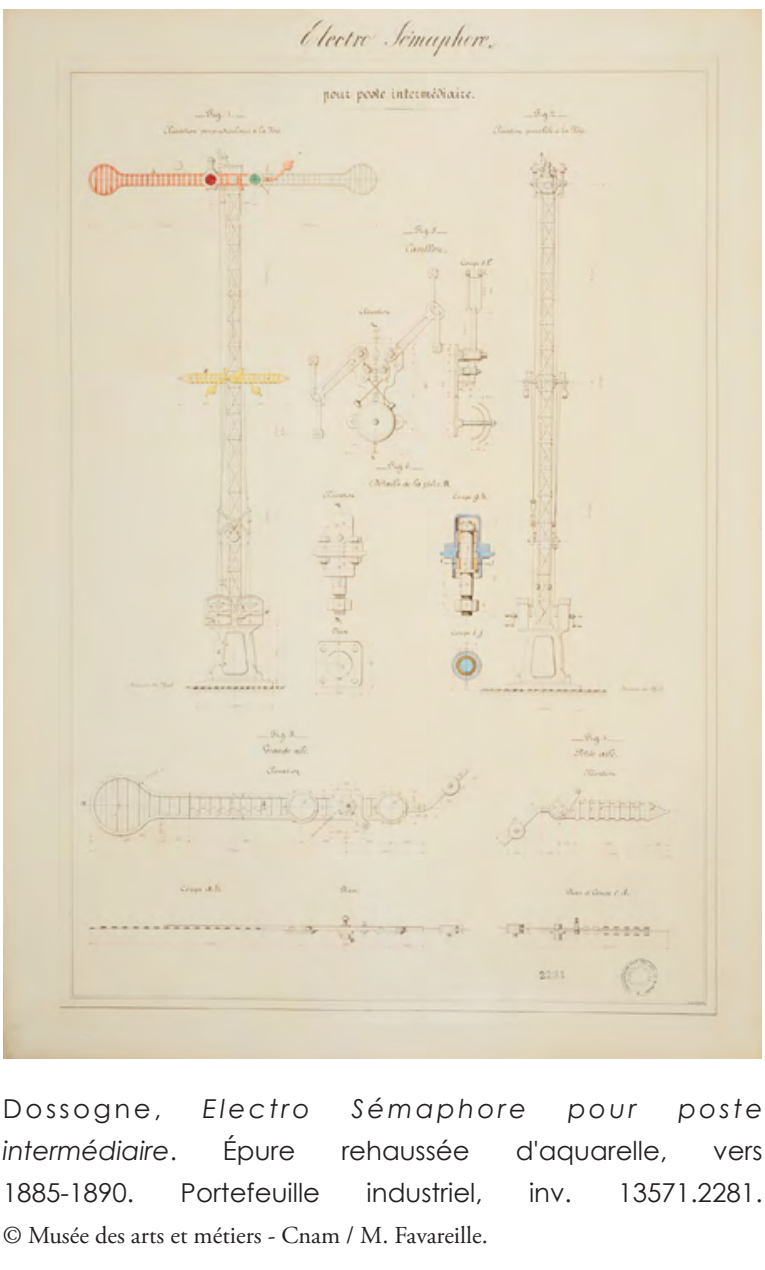

XIXe siècle, les locomotives Crampton (inv. 13767) sont parmi les plus rapides à circuler en France. Un décret de Napoléon III en limite la vitesse à $120 \mathrm{~km} / \mathrm{h}^{36}$. Pourtant, ces machines souffrent de défauts d'adhérence qui vont conduire les ingénieurs à leur préférer des locomotives plus stables mais également plus puissantes, pour remorquer des trains plus longs et plus lourds.

Les quelques modèles possédés par le Conservatoire à l'époque sont souvent entrés par le biais d'acquisitions d'opportunité, comme ce don de M. Deghilage de 1901 qui enrichit les collections de quatre modèles de locomotives (inv. 13411) de la seconde moitié du XIXe siècle : locomotives de type Bourbonnais (030) des chemins de fer de l'Est et du Paris-Orléans, typiques des services

Musée des arts et métiers, La Revue, nº 1, septembre 1992, p. 4-11 ; Gérard Emptoz, "Pierre et Alexandre Clair, constructeurs de modèles ", Musée des arts et métiers, La Revue, $n^{\circ} 3$, mai 1993, p. 4-11 ; Élisabeth Drye, "Les modèles pédagogiques du Musée des arts et métiers ", Musée des arts et métiers, La Revue, n² 28/29, mars 2000, pp. 42-50.

36 Clive Lamming, "La locomotive Crampton 》, Musée des arts et métiers, La Revue, n 15, juin 1996, pp. 15-23. marchandises de l'époque; locomotive de type Columbia (121) des chemins de fer de Paris à Lyon et à la Méditerranée, que l'on vit assurer dans les années 1860-1870 les services voyageurs sur «l'Artère impériale » entre Paris et Lyon; locomotive de type 111, pour les lignes secondaires. À côté de ces modèles déjà dépassés, les visiteurs pourront découvrir la locomotive électrique d'Heilmann, "la fusée " (inv. 13607), et les modernes locomotives de type Atlantic (230), offertes par la Compagnie de l'Ouest (inv. 13563) et de l'Est algérien (inv. 14329). En 1932, l'établissement reçoit une locomotive de type 220 (inv. 16762) qui s'est illustrée sur de nombreuses grandes lignes cinquante ans plus tôt.

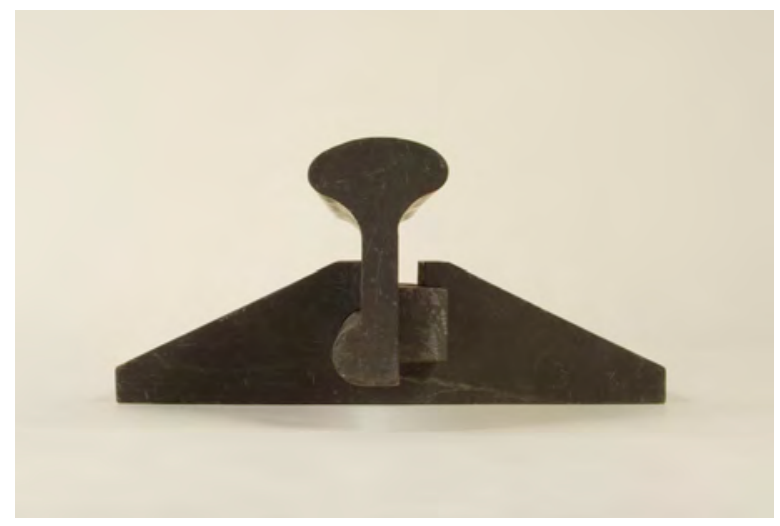

Modèle de rail destiné à la démonstration. II s'agit d'un rail type double champignon avec coussinet. Inv. 05720. (C) Musée des arts et métiers - Cnam / F. Botté.

En 1933, le Conservatoire entre dans une phase d'intense collaboration avec les compagnies ferroviaires. C'est ainsi que la Compagnie du Midi offre une maquette de locomotive de type 231 Pacific, construite par les apprentis des ateliers de Toulouse et de Bordeaux; deux ans plus tard, c'est la Compagnie du Nord qui offre un modèle de sa célèbre Pacific couleur chocolat (inv. 17437), tandis que l'administration des chemins de fer de l'État donne une maquette de ses autorails Bugatti (inv. 17477) en 1937.

\section{Les pièces détachées}

C'est en définitive davantage avec ce que nous pourrions appeler des "pièces détachées " que les innovations ferroviaires de la période entrent dans les collections. Quantitativement, ces éléments sont largement plus nombreux que les modèles "complets". On trouvera ainsi plusieurs pièces provenant de foyers et chaudières, comme des tubes à fumée. On note également la présence de pistons et cylindres, particulièrement intéressants dès lors que l'on se souvient que les 


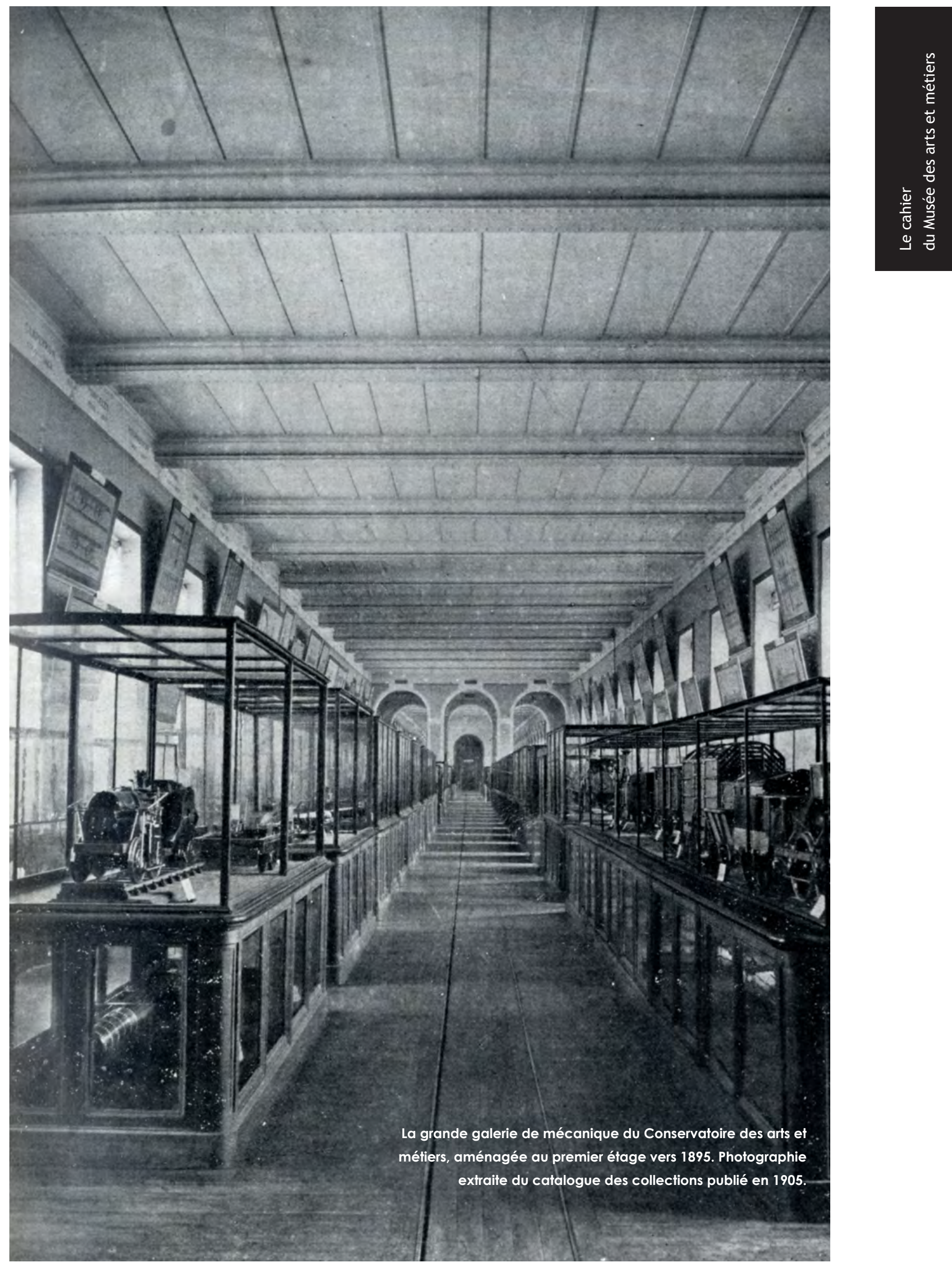




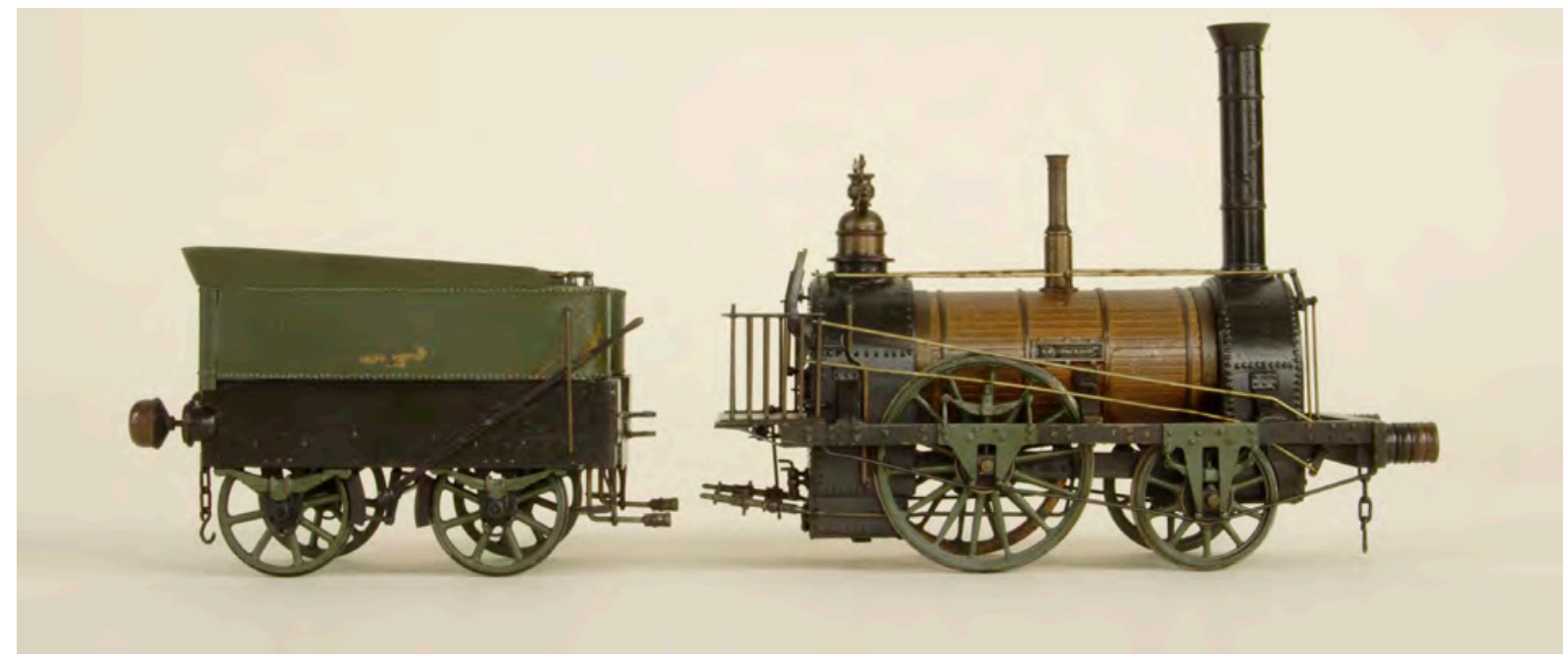

Locomotive Planet 110 « La Jackson ». Modèle au 1/43, vers 1940-1950. Inv. 18653-0001 et 0002. (C) Musée des arts et métiers-Cnam/F. Botté

ingénieurs se penchent sur le compoundage pour optimiser l'utilisation de la vapeur (en la faisant travailler dans un cylindre haute pression puis dans un cylindre basse pression). L'amélioration des cylindres des locomotives est un point crucial pour gagner en puissance et donc en vitesse. Plusieurs pièces permettent d'évoquer l'évolution des freins, avec l'introduction du dispositif Westinghouse (inv. 17426 et 19211 ) qui permet de faire freiner simultanément la locomotive et les wagons.

La voie et les infrastructures

La période 1870-1939 est marquée par une importante modernisation des infrastructures. L'armement des voies devient insuffisant face à un trafic soutenu avec des trains plus lourds. Aux rails en fonte puis en fer seront substitués des rails en acier, plus lourds et plus longs. Les très importants dons de plaques tournantes et de rails, opérés en 1884 (notamment inv. 17180) reviennent sur ces améliorations. De même, les quelque deux cent quinze dessins des appareils de voie et rails de la Compagnie du Midi, donnés par le directeur de ce réseau, nous montrent l'étendue de la fabrication des voies à la fin du XIXe siècle (inv. 13571.2243).

C'est également par le Portefeuille industriel mais aussi par la photographie que les collections s'enrichissent de données quant aux ouvrages d'art qui jalonnent les lignes de chemin de fer. Les séries inv. 10149 et 10241 sont ainsi consacrées à des viaducs ferroviaires, tandis que le célèbre viaduc de Garabit est mis à l'honneur dans la série inv. 10716.
C'est enfin au cours de cette période qu'entrent les quelques éléments qui soulignent la nette amélioration des conditions de sécurité, comme le télégraphe Breguet datant de 1858, qui a accompagné le déploiement du "block système ॥ (voir note infra-paginale 20). Une série de dessins du Portefeuille aborde la sophistication des enclenchements et de la signalisation mécanique avec le dispositif imaginé par l'ingénieur Vignier pour la Compagnie de l'Ouest (inv. 13571.2281).

\section{La salle des chemins de fer de 1944}

Une nouvelle muséographie

Un siècle après la création de la première galerie des chemins de fer, le Conservatoire opère une profonde réorganisation de ses collections ferroviaires. Le projet commence au début de l'année 1942 où Louis Ragey37, directeur du Conservatoire, est contacté par la SNCF pour mettre en place un véritable « "enseignement par l'objet" sur les chemins de fer par la présentation de choses et documents relatifs aux chemins de fer aux enfants des écoles ${ }^{38}$ ). Pour Robert Le Besnerais ${ }^{39}$ et Charles Boyaux ${ }^{40}$, la SNCF profitera d'une section du musée et les maquettes prêtées à l'occasion seront animées par les démonstrateurs. La présentation serait modernisée par les soins de la SNCF au fur et à mesure des progrès techniques. Cette nouvelle partie de

37 Louis Ragey s'est lui-même intéressé aux chemins de fer. II a soutenu un mémoire au début des années 1930 sur La Question du chemin de fer de Bagdad. Voir Jean-Jacques Thiercelain, "Louis Ragey ", in Claudine Fontanon et André Grelon (dir.), op. cit.

38 Procès-verbal du Conseil d'administration du 4 juillet 1942. 39 Directeur général de la SNCF.

40 Directeur commercial de la SNCF. 

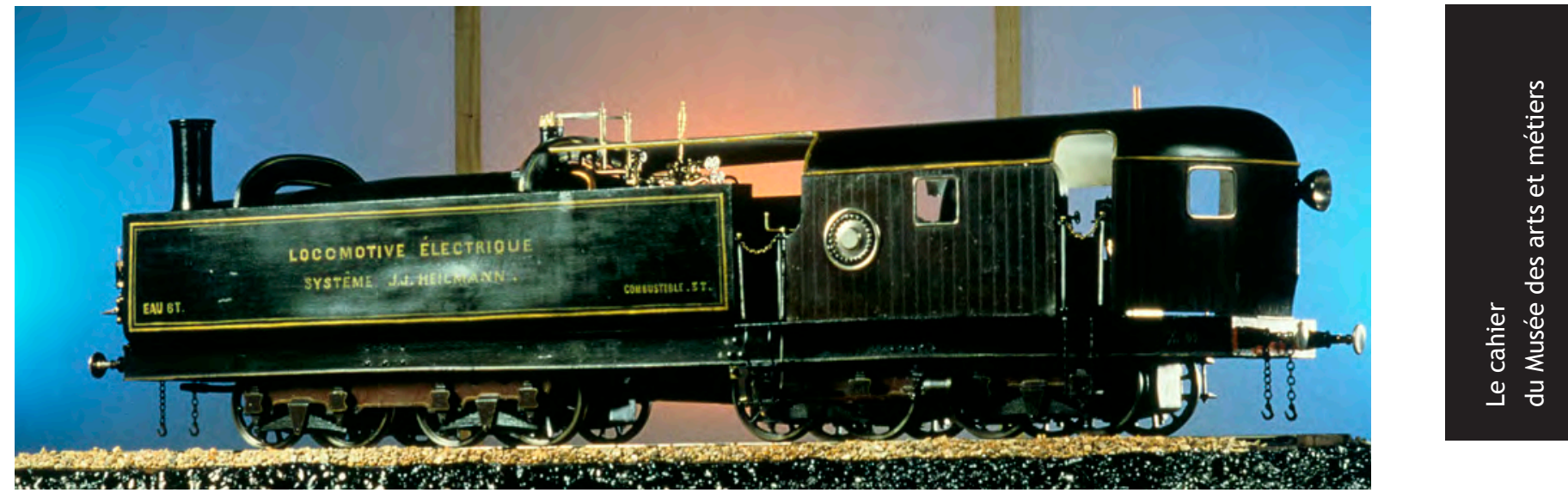

Locomotive électrique "La Fusée ", de JeanJacques Heilmann. Modèle au 1/10, vers 1900. (๑) Studio Photo Cnam.

l'exposition est inaugurée en juin 1944 après des travaux menés conjointement par le Conservatoire et la SNCF ${ }^{41}$.

Les collections ferroviaires, jusqu'alors installées au premier étage, sont déployées au rez-de-chaussée, à proximité de la salle de l'écho. La muséographie de cet espace repose sur des vitrines modernisées, aux arrêtes métalliques. De nombreux objets sont mis en réserve à cette occasion ${ }^{42}$ (dispositifs mécaniques, chaudières, tubes à fumée...) Une quinzaine de tableaux didactiques (aujourd'hui disparus) reviennent en images sur les locomotives et les voitures de voyageurs depuis 1837 (chemin de fer de Saint-Germain-en- Laye) jusqu'à 1937 (inv. 18248 à 18257). II en

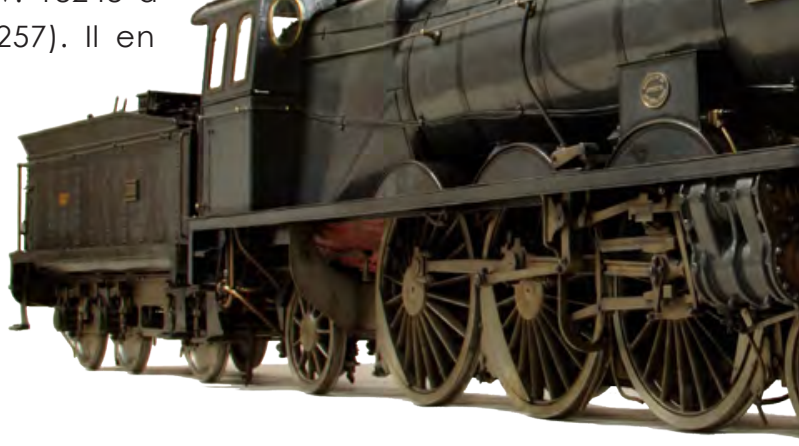

41 Revue générale des chemins de fer, vol. 89, 1970 ; Jean Falaize et Henri Girod-Eymery, À travers les chemins de fer, de l'origine à nos jours, Paris, 1948 ; Procès-verbal du Conseil d'administration du 17 avril 1944.

42 Conservatoire national des arts et métiers. Catalogue du musée, section DB : Transports sur rails, Paris, 1952. Le catalogue des collections établi peu de temps après l'ouverture de la salle des chemins de fer permet de constater la mise en réserves d'une partie des collections de pièces détachées. va de même pour l'évolution des dispositifs de signalisation (inv. 18260), des vitesses commerciales (inv. 18259), ainsi que de l'extension du réseau (inv. 18258). Parallèlement, la salle 53, où étaient présentées les planches du Portefeuille industriel relatives aux chemins de fer, est fermée. Les collections d'arts graphiques demeureront dans les réserves ${ }^{43}$.

Locomotive de type 231 «Pacific » de la compagnie du Paris-Orléans, série 3500, vers 1930. Modèle au 1/10 de 1934 entré en 1944. C) Musée des arts et métiers - Cnam / F B Botté. 


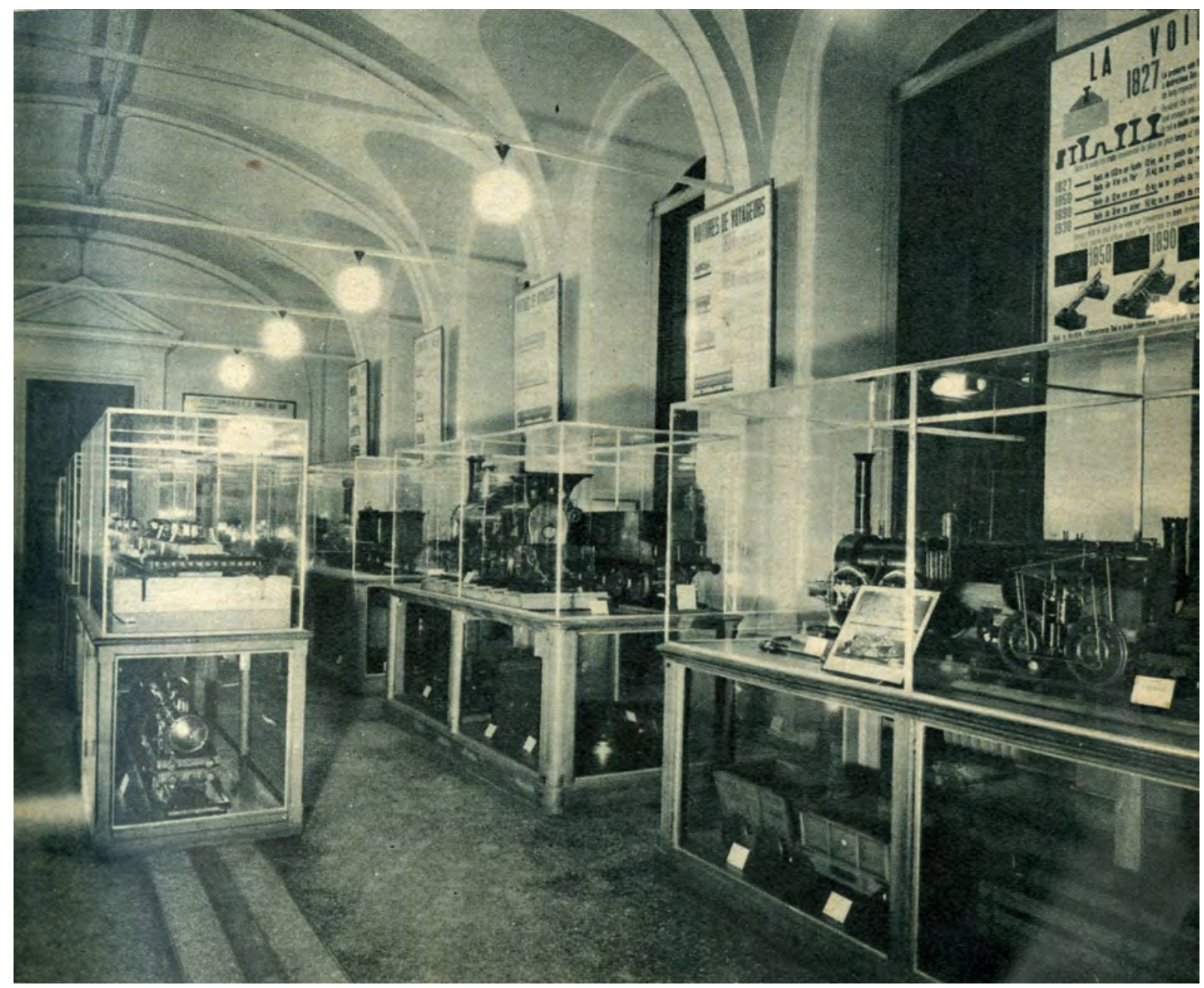

La salle des chemins de fer, inaugurée en juin 1944, au rez-de-chaussée du musée. Photographie extraite de la brochure Le Conservatoire national des arts et métiers, Paris, La Documentation française illustrée. n60, décembre 1951

et de la surchauffe pour tracter plus rapidement des trains dont le poids s'accroît, entre autres par la substitution du métal au bois pour la construction du matériel. On trouve ainsi dans les collections des locomotives qui ont remplacé les Crampton sur les liaisons rapides, comme les locomotives de type 121 de l'ingénieur Forquenot pour le Paris-Orléans (vers 1880, inv. 18165).

Ces locomotives, mises au point à la fin des années 1870, étaient entre autres destinées à la remorque du Paris-Bordeaux, à l'époque le train le plus rapide de France (avec des pointes à $150 \mathrm{~km} / \mathrm{h}$ ) ${ }^{44}$. Viennent ensuite les machines de type 220 de la Compagnie du Nord (vers 1890, inv. 18167) ou du Midi (inv. 18168),

44 Voir «Système du chemin de fer d'Orléans », Bulletin de la Société d'encouragement pour l'industrie nationale, $3^{e}$ série, t. VI, 1879, p. 24. puis les 230 du début du XXe siècle comme celle de la Compagnie de l'Est (vers 1900, inv. 18527). Le règne des célèbres Pacific 231 est évoqué avec un très beau modèle du Paris-Orléans (1934, inv. 18159). Les maquettes montrent également l'évolution du matériel de traction en matière d'énergie (passage de la vapeur au moteur Diesel ou électrique) et en termes de disposition (matériels automoteurs où le train n'est plus composé d'une locomotive et de voitures). On trouve ainsi des locomotives pour les circulations de banlieve (les premières à être électrifiées, avec par exemple l'automotrice à courant continu du Paris-Orléans pour les liaisons entre Paris et Juvisy ${ }^{45}$, Z 4400 - inv. 18161), mais également pour les liaisons grandes lignes (comme dans les Pyrénées avec cette 2D2 5000 du Midi - inv. 18160 ou sur la liaison Paris-Bordeaux avec la 2D2 5546 -inv. 19088).

Une modernisation régulière

Conformément à ce qui était convenu entre le Conservatoire et la SNCF, la présentation a évolvé au fil

45 Bulletin de la Société d'encouragement pour l'industrie nationale, $1^{\text {er }}$ semestre 1911, p. 646. 


\section{Lionel Dufaux}

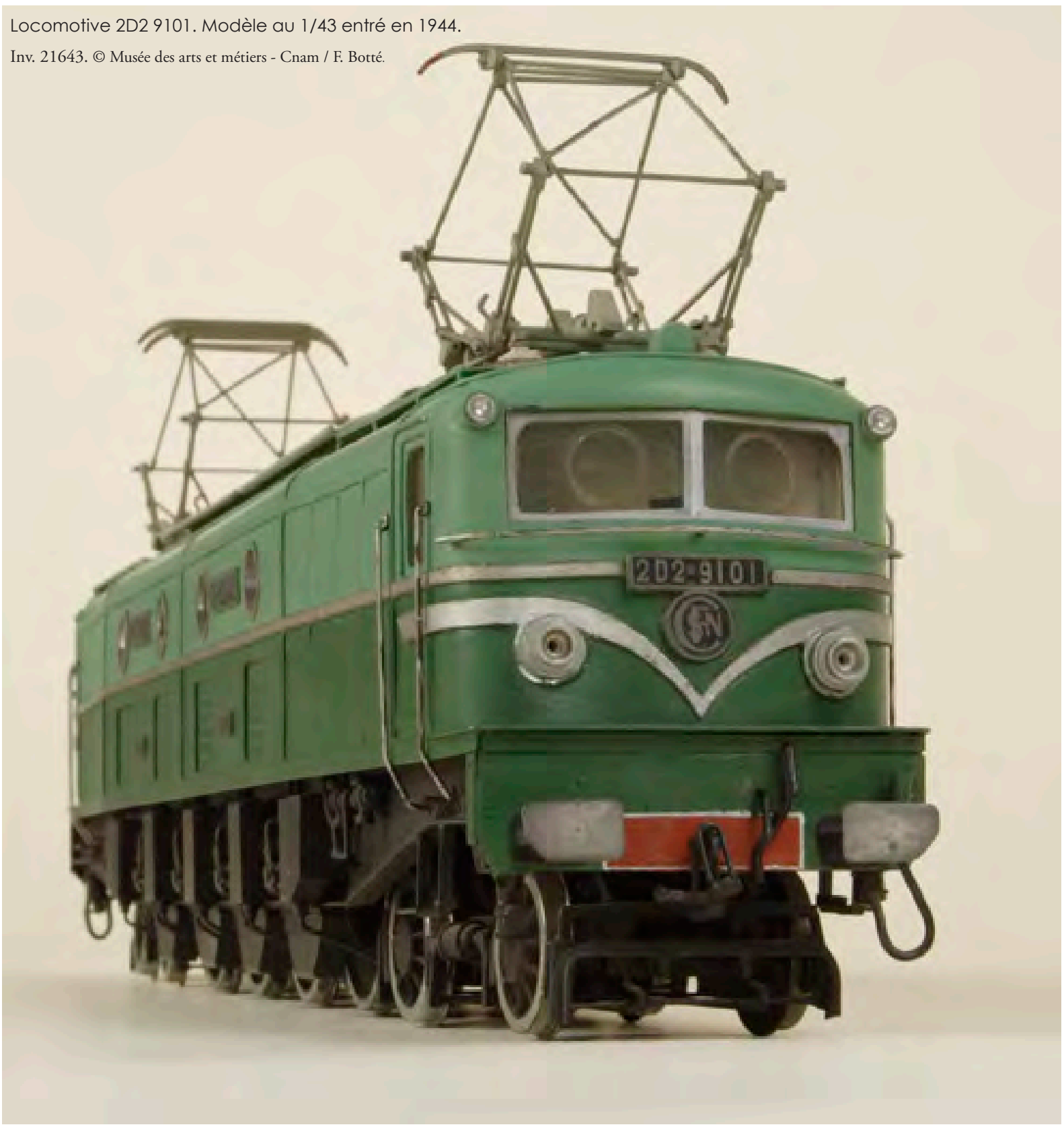

des années ${ }^{46}$. Six objets intègrent les collections en 1945, neuf en 1946, neuf encore en 194747, vingt-cinq en 194848, quatorze en 1949, trois en 1952, un objet déposé en 1959, quatre en 1960, deux en 1961, vingt-deux en 196449, un en 1965, deux en 1969, un en 1970, dix en 1972 et deux en 1975. Entre-temps, quarante-cinq objets entrés en 1944 sont radiés des collections entre 1946 et 1965. II s'agit,

46 Données extraites de la main courante du musée et croisées avec le catalogue de 1952. Merci à Sandra Delaunay, responsable des collections "Transports », et à Élisabeth Lefèvre, du service de l'Inventaire, pour leur aide et leurs précieux conseils quant à ce prêt de la SNCF.

47 Procès-verbal du Conseil d'administration du 11 avril 1947. 48 Procès-verbal du Conseil d'administration du 10 décembre 1948. 49 Procès-verbal du Conseil d'administration du 18 décembre 1964. pour la plupart, d'objets anecdotiques et décoratifs dont l'intérêt semble limité, comme une série de vues panoramiques, des assiettes décorées ou un mouchoir imprimé.

Quelque cinquante nouvelles maquettes intègrent le Conservatoire et illustrent la modernisation des chemins de fer français. II faut rappeler que l'image des trains en France reste assez archaïque à l'époque ; le chemin de fer subit de plein fovet la concurrence de l'automobile particulière et le développement des liaisons aériennes intérieures (Air Inter est créée en 1957). La SNCF s'emploie alors à changer l'image du train, notamment par des campagnes publicitaires audacieuses (confiées entre autres à Salvador Dalí) ${ }^{50}$.

50 Lionel Dufaux, « Notices » in Toujours plus vite ! Les défis du rail, op. cit., p. 123. 


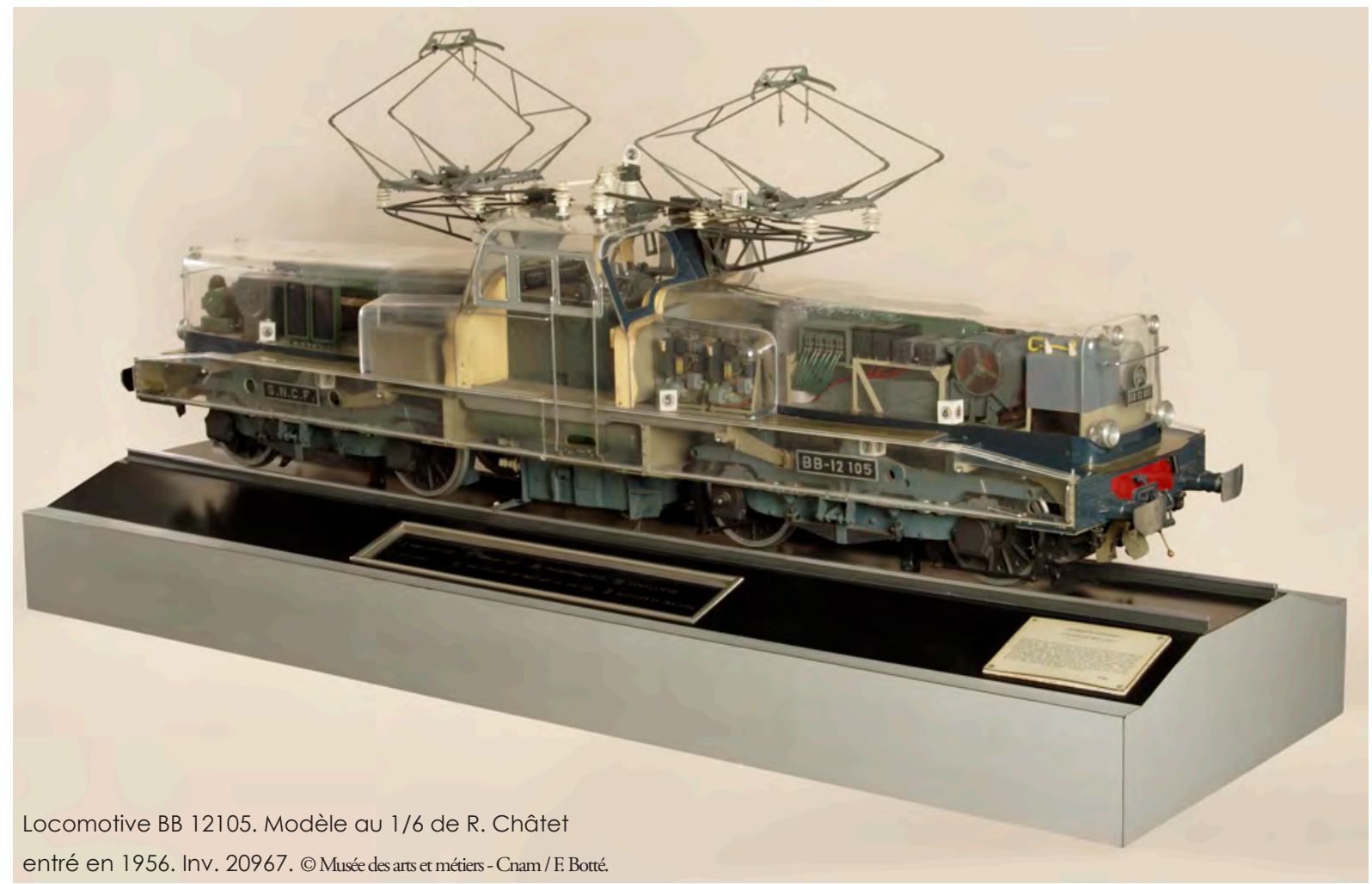

On peut penser que c'est dans le même esprit que la présentation de la salle des chemins de fer est reprise. Désormais, outre les maquettes historiques des anciens réseaux, on trouvera des locomotives symboles de l'électrification du réseau en courant continu (2D2 9100, inv. 21643) et en courant monophasé (BB 12000, inv. 21641), des autorails pour les liaisons transversales (inv. 21647) ou encore les rames à grand parcours pour les relations internationales sous le label «Trans Europ Express » (inv. 21648).

Le caractère didactique de la présentation est toujours en vigueur, notamment avec la cabine de conduite d'une BB 16000 à l'échelle (inv. 21882) ou la maquette en plexiglas au 1/6 de la BB 12100 (inv. 20967) qui offre aux visiteurs l'occasion de découvrir les rincipaux organes d'une locomotive électrique.

Les dernières maquettes entrées dans ce cadre sont celles du turbotrain (inv. 22246), premier engin ferroviaire motorisé avec une turbine à gaz inspirée des moteurs d'hélicoptères. II sera rejoint en 1973 par la maquette du TGV 001, rame expérimentale du train à grande vitesse, également équipée d'une turbine à gaz et détentrice du record mondial de vitesse sur rails en traction thermique (318 km/h en décembre 1972).

Cet enrichissement considérable des collections ferroviaires pose des problèmes de place, et Maurice Daumas, conservateur du musée entre 1960 et 1976 , expose les choix opérés dans les années 1960 :
« Pour la section des Chemins de fer, nous avons demandé aux équipes qui ont étudié le plan de réinstallation de concevoir des meubles dont la partie basse permettait de mettre en réserve les maquettes de locomotives et de wagons présentant un intérêt tout à fait secondaire. Ainsi les locomotives à vapeur qui occupaient presque toute la totalité de la salle dans l'ancienne installation ont pu être regroupées sur la moitié de la surface seulement, laissant disponibles des espaces pour traiter de la traction électrique et de la traction diesel, c'est-à-dire des moyens de traction moderne des chemins de fer ${ }^{51}$. ı

Maurice Daumas indique que le réaménagement de la salle des chemins de fer a été étudié par des équipes formées d'élèves d'une chaire du Conservatoire ${ }^{52}$. Peutêtre s'agit-il de la chaire d'arts appliqués aux métiers, dont Jean Prouvé était le professeur titulaire dès 1958 ? Daumas recherchait une plus forte coopération entre les services du musée et les professeurs de l'établissement.

\section{La mise en sommeil des collections}

Les collections du Conservatoire entrent, au début des années 1970, dans une certaine léthargie, au moment où va se construire le premier train à grande vitesse. L'ouverture du musée français du Chemin de

51 Maurice Daumas, art. cit.

$52 \mathrm{lbidem}$. 

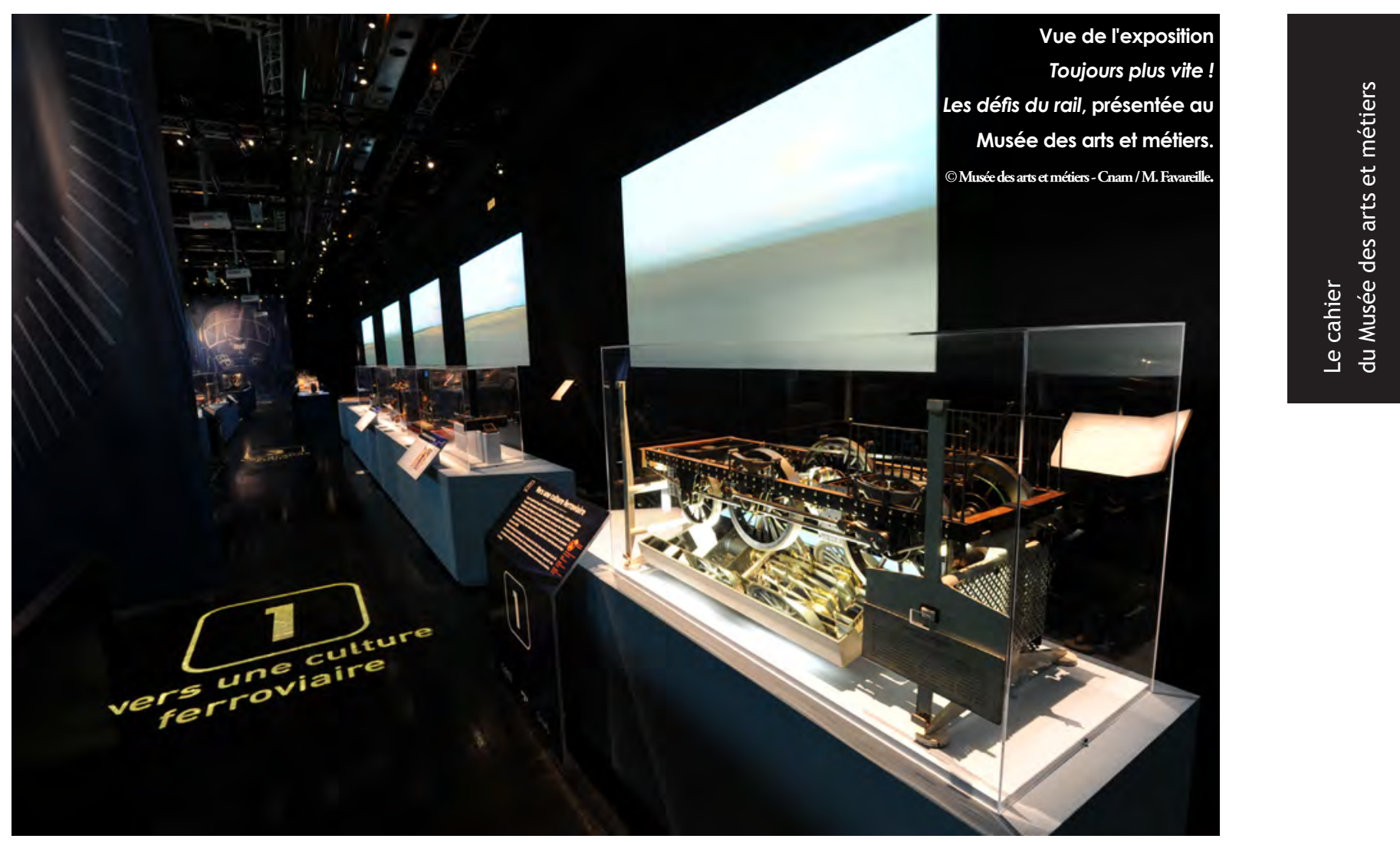

fer (actuelle Cité du train) à Mulhouse, en 1971, peut en partie l'expliquer ${ }^{53}$. La profonde rénovation du Musée des arts et métiers, entre 1992 et 2000, va conduire à la disparition de la salle des chemins de fer. Désormais redéployées dans un "domaine Transports" installé dans l'escalier d'honneur, la salle de l'écho et l'ancienne église Saint-Martin-des-Champs, les collections ferroviaires sont mises en relation avec les autres modes de transport (automobile, navigation et aviation). La très grande majorité des pièces sont alors conservées dans les réserves du musée, à Saint-Denis, où elles restent visibles pour être étudiées par les chercheurs.

La dernière entrée massive de maquettes ferroviaires date de 1993. II s'agit de la dation (inv. 40942 et 40943) opérée dans le cadre de la succession Paul Arzens (1903-1990). Ce designer, connu pour

53 || semble que la question de la préservation du matériel ancien se soit posée dès 1944 à la SNCF. En 1961, elle entrepose des locomotives et des voitures au dépôt de Châlonssur-Marne dont l'Association française des amis du chemin de fer publiera le catalogue (1965). La même année, la Société industrielle de Mulhouse crée un comité chargé d'étudier la possibilité de créer un musée ferroviaire. Par décision du 6 juin 1969 du ministre des Transports, la SNCF est autorisée à se rapprocher de la Sim pour créer le musée français du Chemin de fer par le biais d'une association (loi 1908 type Alsace-Moselle). ses créations dans le domaine de l'automobile, a été à l'origine de nombreux matériels pour la SNCF. II imagine ainsi, dans les années 1950, des locomotives aux postes de conduite arrondis. Les BB 9200 (inv. 40943-0001 à 0005), 16000 (inv. 40943-0006) ou 25000 en seront, à partir de 1957, de parfaites illustrations. À partir des années 1970, Arzens conçoit des formes plus agressives, avec la génération des " nez cassés ॥, parmi lesquelles les CC 6500 (" reines de l'Artère impériale » entre Paris et Marseille), les BB 15000 ou les CC 40100 (inv. 40943-0008).

Les nombreux modèles entrés au Conservatoire montrent la manière dont Paul Arzens travaillait. Le designer se servait de maquettes à l'échelle HO (1/43) achetées dans le commerce pour expérimenter de nouvelles livrées. On peut ainsi voir sur les nombreuses voitures les traces de peinture qui nous laissent supposer que les modèles ont servi à Paul Arzens à affiner la livrée dite "Trans Europ Express ॥, utilisée sur les voitures " Grand Confort» (inv. 40943-0037 à 0040) employées sur le "Capitole" (Paris-Toulouse) ou "l'Aquitaine" (Paris-Bordeaux). C'est en travaillant sur le matériel en Inox de type "Mistral 69 ॥, roulant sur les prestigieux trains de "l'Artère impériale » ou entre Paris et la Belgique, qu'Arzens a imaginé la livrée des « Turbotrains ॥ (inv. 40943-0027 à 0032). 
Les collections ferroviaires du Musée des arts et métiers se sont constituées en suivant la naissance et le développement du réseau des chemins de fer français. Là où la Cité du Train de Mulhouse ou le musée national de la Voiture et du Tourisme au château de Compiègne présentent du matériel réel préservé, le musée du Conservatoire a privilégié les modèles de démonstration, les pièces détachées et les images. Cette spécificité fait toute l'originalité de la collection ferroviaire - la première collection institutionnelle française dans ce domaine. La poursuite des investigations dans les archives de l'établissement permettra de démontrer si les collections ont effectivement été réunies pour servir de support à l'enseignement ou si elles ont fait l'objet d'expériences dans les domaines de la mécanique et de la thermodynamique.

Il semble toutefois avéré qu'entre la fin des années 1830 et les années 1930, ces collections ont été réunies comme des exemples significatifs d'applications de la mécanique et de la thermodynamique. Cette orientation changera radicalement à partir de 1944, où l'axe historique sera privilégié pour l'enrichissement et la présentation des collections. Les choix opérés à l'époque ont fait entrer dans les collections des modèles de matériel ferroviaire de grande qualité, où les notions de vitesse, de sécurité et de confort étaient généralement prédominantes. De nouveaux axes pourraient aujourd'hui être développés, comme celui de la très grande vitesse ferroviaire où la France a été pionnière. À la suite de l'exposition Toujours plus vite! Les défis du rail, plusieurs pièces détachées pourraient ainsi entrer dans les collections (rove et freins de TGV), renouant en partie avec la tradition née au XIXe siècle au Conservatoire. De la même manière, des pièces illus-trant l'histoire des techniques ferroviaires à l'étranger pourraient prendre place dans les collections en offrant aux visiteurs une comparaison de l'évolution technique en s'intéressant aux choix faits en Grande-Bretagne, aux États-Unis ou au Japon, par exemple. 